\title{
$\angle S$ Research Square \\ Identification of hub genes and potential drugs in hepatocellular carcinoma through integrated bioinformatics analysis
}

\section{Xiaolong Chen}

The First Affiliated Hospital of Chongqing Medical University

\section{Zhixiong Xia}

The Center Hospital of Wuhan

\section{Yafeng Wan}

Daping Hospital, Army Medical University

Ping Huang ( $\nabla$ huangpchina@sina.com )

The First Affiliated Hospital of Chongqing Medical University https://orcid.org/0000-0002-0221-8551

\section{Primary research}

Keywords: hepatocellular carcinoma, differently expressed genes, bioinformatics analysis, hub genes, drug

Posted Date: October 14th, 2020

DOI: https://doi.org/10.21203/rs.3.rs-88832/v1

License: (9) (1) This work is licensed under a Creative Commons Attribution 4.0 International License. Read Full License 


\section{Abstract}

\section{Background}

Hepatocellular carcinoma (HCC) is the third cancer-related cause of death in the world. Until now, the involved mechanisms during the development of HCC are largely unknown. This study aims to explore the driven-genes and potential drugs in HCC.

\section{Methods}

Three mRNA expression datasets were used to analyze the differentially expressed genes (DEGs) in HCC. The bioinformatics approaches include identification of DEGs and hub genes, GO terms analysis and KEGG enrichment analysis, construction of protein-protein interaction network. The expression levels of hub genes were validated based on TCGA, GEPIA and the Human Protein Atlas. Moreover, overall survival and disease-free survival analysis of the hub genes were further conducted by Kaplan-Meier plotter and the GEPIA. DGIdb database was performed to search the candidate drugs for HCC.

\section{Results}

Finally, 197 DEGs were identified. The PPI network was constructed using STRING software. Then ten genes were selected and considered as the hub genes. The ten genes were all closely related to the survival of HCC patients. DGIdb database predicted 39 small molecules as the possible drugs for treating HCC.

\section{Conclusions}

Our study provides some new insights into HCC pathogenesis and treatments. The candidate drugs may improve the efficiency of HCC therapy in future.

\section{Introduction}

Hepatocellular carcinoma (HCC) is one of major health problems worldwide [1, 2]. It affects more than half a million people worldwide every year, with about $30 \%$-year survival rate $[3,4]$. Although a variety of therapies have been used to treat HCC in the past few decades, the treatment effect is still unsatisfactory due to postoperative recurrence and drug resistance. Increasing evidence has showed that the molecular pathogenesis of HCC may be closely associated with living environment and genetic factors, such as P53 inactivation, several oncogene activation and gene mutation $[5,6]$. However, the precise mechanisms underlying $\mathrm{HCC}$ development and progression remain unclear.

Recently, the rapid development of high-throughput RNA microarray analysis, has allowed us to better understand the underlying mechanisms and general genetic alterations involved in HCC occurrence and metastasis. RNA microarrays have been extensively applied to explore HCC carcinogenesis through gene expression profiles and identification of altered genes $[7,8,9]$. Meanwhile, many large public databases 
such as The Cancer Genome Atlas (TCGA), and Gene Expression Omnibus (GEO) can be performed to screen the differentially expressed genes (DEGs) from microarray data related to the initiation and progression of HCC.

Most of HCC patients have a relatively long latent period, therefore many HCC patients are in the intermediate or advanced stage when first diagnosed, in which radical surgery is no longer desirable [10]. However, many chemotherapy are often with unsatisfactory curative effects and some severe sideeffects. For an example, sorafenib shows a 3-month median survival benefit, but is related to two grade 3 drug-related adverse events namely diarrhea and hand-foot skin reaction [11]. At present, the diseasefree survival (FDS) and overall survival (OS) of HCC patients remained relatively short, highlighting the importance of developing new drugs.

In the study, Three mRNA expression profles were downloaded (GSE121248 [12], GSE64041 [13], and GSE62232 [14]) from GEO database to identify the genes correlated to HCC progression and prognosis. Integrated analysis included identifying DEGs using the GEO2R tool, overlapping three datasets using a Venn diagram tool, GO terms analysis, KEGG biological pathway enrichment analysis, PPI network construction, hub genes identification and verification, construction of hub genes interaction network, survival analysis of the screened hub genes and exploration of the candidate small molecular drugs for HCC.

\section{Materials And Methods}

\section{Data collection}

HCC and adjacent normal tissue gene expression profiles of GSE 121248, GSE64041, and GSE62232 were downloaded from GEO database (http://www.ncbi.nlm.nih.gov/geo/) [15]. The microarray data of GSE121248 was based on GPL571 Platforms (Affymetrix Human Genome U133 Plus 2.0 Array) and included 70 HCC tissues and 37 normal tissues (Submission date: Oct 15, 2018). The GSE64041 data was based on GPL6244 Platforms (Affymetrix Human Gene 1.0 ST Array) and included 60 biopsy pairs from HCC patients, 5 normal liver biopsies (Submission date: Dec 10, 2014). The data of GSE62232 was based on GPL571 Platforms (Affymetrix Human Genome U133 Plus 2.0 Array) and included 81 HCC cancer tissues and 10 normal liver tissues (Submission date: Oct 09, 2014). The above datasets meet the following criteria: (1) they used tissue samples from human HCC tissues and adjacent or non-tumor liver tissues; (2) each dataset involved more than 90 samples.

\section{DEGs identification}

GEO2R (https ://www.ncbi.nlm.nih.gov/geo/geo2r/) was used to screen the DEGs in HCC tumor tissues and non-tumor liver tissues [16]. Adjusted P-values (adj. P) $<0.05$ and $|\log F C|>1$ were set as the cut-off criterion to select DEGs for every dataset microarray respectively [17]. Then, the overlapping DEGs among the three datasets was identified by Venn diagram tool (http://bioin fogp.cnb.csic.es/tools/venny/). Visual hierarchical cluster analysis was also performed to display the volcano plot of DEGs. 


\section{GO and KEGG pathway enrichment analysis}

To explore the functions of DEGs, Enrichr database was performed to conduct $\mathrm{GO}$ terms analysis and KEGG pathway enrichment analysis [18]. We submitted the DEGs, including 54 upregulated genes and 143 downregulated genes, into Enrichr database. The GO terms consisted of following three parts: biological process (BP), cellular component (CC) and molecular function (MF). Adj. $\mathrm{P}<0.05$ was regarded as statistically significant.

\section{Construction of PPI network and screening of hub genes}

PPI networks are the networks of protein complexes due to their biochemical or electrostatic forces. The Search Tool for the Retrieval of Interacting Genes (STRING) (https://string-db.org/cgi/input .pl/) is a database constructed for analyzing the functional proteins association networks [19]. The screened DEGs had been submitted to the STRING database, and all PPI pairs with a combined score of $>0.4$ were extracted. The degree of all nodes was calculated by Cytoscape (v3.6.1) plugin cytoHubba [20]. In the study, the genes with the top 10 highest degree values were regarded as hub genes.

\section{Validation of hub genes}

To validate the mRNA expression level of the hub genes in HCC, The GEPIA database was used to show difference in the mRNA expression level of each hub gene between liver hepatocellular carcinoma (LIHC) and non-cancerous liver samples [21]. Afterwards, the protein expression levels of the hub genes in normal and HCC tissues were visualized through the Human Protein Atlas (HPA) database that contains immunohistochemistry-based expression data for about 20 common types of cancers [22].

\section{Genetic alterations of hub genes}

The LIHC dataset (TCGA, PanCancer Atlas ) including the data of 348 samples was selected for the analysis of the genetic alterations in hub genes using cBioPortal database. This database allows for visualization, analysis and downloading of a lot of cancer genomic dataset [23]. The genomic alterations included gene mutations, copy number variations, deep deletion, mRNA expression z-scores (RNA Seq V2 RSEM) with a z-score threshold of \pm 2.0 and protein expression z-scores. According to the online instructions of cBioPortal, the analysis on disease-free survival (DFS) and overall survival (OS) were also carried out.

\section{Survival analysis for hub genes}

Kaplan-Meier plotter is extensively applied to explore the roles of more than 54,000 genes in OS based on 13,316 tumor samples from GEO, EGA and TCGA datasets including 364 patients with liver cancer. The relation between $\mathrm{OS}$ and hub genes expressed in patients with liver cancer was determined by the KaplanMeier survival analysis [24]. Moreover, the relation between DFS and the genes expressed in LIHC patients was explored through the online tool GEPIA database. The lower and upper $50 \%$ of gene expression were set as the standard for analysis. In the present study, HCC patients were devided into 2 groups based on 
the median expression values of the hub genes. Log-rank $\mathrm{P}<0.01$ were regarded as statistically significant.

\section{Drug-hub gene interaction}

The screened hub genes were also regarded as the promising targets for searching drugs through the DGIdb database (http://dgidb.genome.

wustl.edu/) [25]. This database have drug-gene interaction data from 30 disparate sources such as ChEMBL, DrugBank, Ensembl, NCBI Entrez, PharmGKB, and literature in NCBI PubMed. Drugs supported by no less than one databases or PubMed references were validated as the potential drugs. The final list only contained the drugs that have been approved by the Food and Drug Administration (FDA). Additionally,the identified target gene network was constructed through STITCH database (http://stitch.embl.de/), a software which also incorporated drug-gene relationships [26, 27].

\section{Results}

\section{Identification of DEGs}

According to GSE121248 dataset analysis, 943 DEGs were successfully identified, including 325 upregulated and 618 downregulated genes. For GSE64041 dataset, 289 DEGs were observed, including 87 upregulated and 202 downregulated genes. For GSE62232 dataset, 1,355 DEGs were identified, involving 817 upregulated and 538 downregulated genes. Venn analysis was performed to examine the intersection among the three DEGs profiles. Then, 197 DEGs were identified from the three profile datasets (Table 1). Obviously, 54 DEGs were significantly upregulated (Fig. 1A), while 143 DEGs were markedly downregulated (Fig. 1B) in HCC tissues. These 197 DEGs were plotted in Fig. 1C, where the red and green dots represented the upregulated and downregulated DEGs, respectively. In addition, the mRNA expression level of these 197 DEGs was visualized in the form of a heatmap using data profile GSE20347 (Additional file 1:Figure S1). 
Table 1

The common DEGs of three gene expression profiles (adj. Pval. $<0.05,|\log F C|>1.0$ )

\section{DEGs Gene symbol}

Upregulated

SPINK1; TPX2; EDIL3; ASPM; FLVCR1; AKR1B10; GINS1; SRXN1; KPNA2; ANLN; NQ01; FOXM1; EZH2; CCNB2; RBM24; PRC1; CDK1; TOP2A; TXNRD1; SPARCL 1; CDC6; FAM72A; MAP2; AURKA; BUB1B; DLGAP5; NMRAL1P1; LEF1; MKI67; CAP2; DTL; GPC3; CCL20; ROB01; SPP1; SQLE; KIF20A; UBD; RRAGD; CD200; ITGA6; LCN2; MELK; SLC7A11; ITGA2; CCNA2; CDKN3; BUB1; NUF2; NCAPG; UBE2T; CENPF; NUSAP1; ECT2

Downregulated

$(143)$

\begin{abstract}
TUBE1; BBOX1; XDH; SDS; CXCL14; IGF1; DPT; CYP39A1; SLC25A47; PROZ; C8A; ZG16; MBL2; SLC10A1; SLCO1B3; PRG4; CYP1A2; UROC1; FCGR2B; F9; BCO2; ACSM3; CYP2C19; C3P1; LPA; CD5L; GHR; CLEC1B; TAT; LIFR; BHMT; COLEC10; VNN1; LYVE1; STEAP3; SHBG; DNASE1L3; ALDH8A1; NAT2; C7; BCHE; SAA2-SAA4; AKR1D1; CXCL12; GNMT; C1 orf168; GPD1; CRHBP; EHD3; WDR72; IDO2; BDH2; CYP3A43; SLC38A4; DBH; FBP1; ADH4; OIT3; MT1M; SLC39A5; CETP; SRD5A2; ADRA1A; PBLD; SRPX; CYP4A22; KLKB1; GNA01; ENO3; MT1G; SLC19A3; PGLYRP2; TENM1; INS-IGF2; CYP2C8; STEAP4; IL13RA2; SPP2; IGHM; MT1F; FETUB; MFSD2A; HHIP; APOA5; CYP2B7P; KCND3; PPP1R3B; LY6E; ITGA9; OLFML3; CNDP1; FCN3; GBA3; PDGFRA; CLEC4G; PHGDH; CYP2B6; CCBE1; FXYD1; PCK1; KMO; ANK3; CLRN3; MT1H; CLEC4M; NPY1R; ESR1; TDO2; VIPR1; IGFBP3; PLAC8; HAMP; DCN; IL1RAP; RDH16; CYP8B1; TMEM27; AFM; HPGD; LPAL2; THRSP; CYP4A11; STAB2; HGFAC; ADGRG7; OGDHL; PZP; SLCO4C1; FREM2; BMPER; AADAT; GPM6A; HGF; MOGAT2; CYP3A4; EPHX2; GLS2; HABP2; APOF; ANGPTL1; PTGIS; GRAMD1C; SLC7A2
\end{abstract}

Figure 1 Identification of common DEGs from GSE121248, GSE64041, and GSE62232 datasets. Venn diagram of (A) upregulated and (B) downregulated DEGs based on the three GEO datasets. (C) Volcano plot of the 197 DEGs.

Red, upregulation; green, downregulation. The intersecting areas represent

the commonly altered DEGs. The t-test was used to analyze DEGs, with the

cut-off criteria of $|\log F C|>1.0$ and adj. P $<0.05$. DEGs, differentially expressed genes; GEO, Gene Expression Omnibus; logFC, log-fold change.

\section{Functional enrichment analysis of DEGs}

GO annotation and KEGG pathways enrichment analysis were conducted through Enrichr database(http://amp.pharm.mssm.edu/Enrichr/). Figure 2 showed the top 10 enriched GO terms and KEGG pathways. As shown in Fig. 2A, GO BP analysis revealed that these 197 DEGs were significantly enriched in epoxygenase P450 pathway, steroid metabolic process, kynurenine metabolic process and arachidonic acid metabolic process. The top four significantly enriched CC terms included condensed nuclear chromosome, centromeric region, spindle, integral component of plasma membrane, and condensed nuclear chromosome kinetochore (Fig. 2B). For GO MF analysis, the top four significantly enriched terms were oxidoreductase activity, steroid hydroxylase activity, oxidoreductase activity, heme binding (Fig. 2C). Additionally, the top four markedly enriched pathways for these 197 DEGs were Retinol metabolism, Arachidonic acid metabolism, Tryptophan metabolism and Caffeine metabolism (Fig. 2D). 
Figure $2 \mathrm{GO}$ annotation and KEGG pathway enrichment analysis of 197 DEGs. The top 10 enriched GO (A) BP, (B) CC, (C) MF terms and (D) KEGG

pathways. GO, Gene Ontology; KEGG, Kyoto Encyclopedia of Genes and Genomes; DEGs, differentially expressed genes; $\mathrm{BP}$, biological process; $\mathrm{CC}$, cellular component; $\mathrm{MF}$, molecular function.

\section{PPI network construction and hub genes identification}

The STRING database was performed to determine the PPI network among

the 197 DEGs. The PPI network including 197 nodes (genes) and 968 edges (interactions) was constructed through STRING database (Additional file 1:Figure S2). The PPI enrichment $p$-value $<1.0 \times$ $10^{-16}$. Ten genes with the highest degree scores were reged as the hub genes by applying the Cytoscape (v3.6.1) plugin cytoHubba. The results revealed that forkhead box M1(FOXM1) was the hub gene with the highest connectivity degree, followed by aurora kinase A (AURKA), cyclin A2 (CCNA2), cyclin-dependent kinase inhibitor 3 (CCKN3), marker of proliferation Ki-67 (MKI67), enhancer of zeste 2 polycomb repressive complex 2 subunit (EZH2), cell division cycle 6 (CDC6),cyclin-dependent kinase 1 (CDK1),cyclin B1 (CCNB1),Topoisomerase (DNA) II alpha (TOP2A) (Table 2).

Using cytoHubba software,the PPI network of the screened 10 hub genes were constructed, which had a strong interaction among each other (Fig. 3A). The interaction network of 10 hub genes and their related genes was also identified by the FunRich software (Fig. 3B) [28]. The hub genes and their related genes could be enriched in many biological pathways through the enrichment functions of FunRich tool. KEGG analysis established that markedly enriched pathways for the hub genes included progesterone mediated oocyte maturation, cell cycle, cellular senescence, oocyte meiosis, p53 signaling pathway, Viral carcinogenesis, Lysine degradation, and gap junction (Fig. 3C). 
Table 2

Top ten hub genes with higher degree of connectivity.

\begin{tabular}{|lll|}
\hline Gene symbol & Gene description & Degree \\
\hline FOXM1 & Forkhead box M1 & 36 \\
\hline AURKA & Aurora kinase A & 34 \\
\hline CCNA2 & Cyclin A2 & 34 \\
\hline CDKN3 & Cyclin-dependent kinase inhibitor 3 & 34 \\
\hline MKI67 & Marker of proliferation Ki-67 & 34 \\
\hline EZH2 & Enhancer of zeste 2 polycomb repressive complex 2 subunit & 33 \\
\hline CDC6 & Cell division cycle 6 & 33 \\
\hline CDK1 & Cyclin-dependent kinase 1 & 33 \\
\hline CCNB1 & Cyclin B1 & 33 \\
\hline TOP2A & Topoisomerase (DNA) II alpha & 33 \\
\hline
\end{tabular}

Figure 3 Interaction network and KEGG analysis of the hub genes. (A) The top 10 hub genes in the PPI network were screened by Cytoscape (v3.6.1) plugin cytoHubba. The 10 hub genes are displayed from red (high degree value) to yellow (low degree value). (B)The PPI network of the 10 hub genes and their related genes, created by the FunRich software. (C) KEGG pathway enrichment analysis of the 10 hub genes. PPI, protein-protein interaction; STRING, search tool for the retrieval of interacting genes; KEGG, Kyoto encyclopedia of genes and genomes.

\section{Validation of mRNA expression levels of these 10 hub genes in HCC}

First, a differential analysis on the mRNA expression levels of FOXM1, AURKA, CCNA2, CCKN3, MKI67, EZH2, CDK1, CCNB1, and TOP2A, between HCC and non-tumor liver tissues was conducted through the GEPIA database.

As showed in Fig. 4, the mRNA expression levels of (Fig. 4A) FOXM1, (Fig. 4B) AURKA, (Fig. 4C) CCNA2, (Fig. 4D) CCKN3, (Fig. 4E) MKI67, (Fig. 4F) EZH2, (Fig. 4G) CDC6, (Fig. 4H) CDK1, (Fig. 4I)CCNB1, and (Fig. 4J) TOP2A were significantly upregulated in HCC tissues $(P<0.01)$ compared to those in normal liver tissues. Hierarchical clustering analysis also performed that the mRNA expression levels of all the hub genes were significantly increased in primary hepatic cancer (TCGA Live Cancer, $n=469$ ) tissues compared to non-tumor tissue samples through UCSC Xena Browser (Additional file 1:Figure S3). These findings were consistent with the obtained GEO microarray data.

Figure 4 Validation of the mRNA expression levels of (A) FOXM1, (B) AURKA, (C) CCNA2, (D) CCKN3, (E) MKI67, (F) EZH2, (G) CDC6, (H) CDK1, 
(I) CCNB1, and (J) TOP2A in LIHC tissues and normal liver tissues using GEPIA database. These ten box plots are based on 369 LIHC samples (marked in red) and 160 normal samples (marked in gray). *P< 0.01 was considered statistically significant. LIHC, liver hepatocellular carcinoma.

\section{Validation of protein expression levels of these 10 hub genes in HCC}

The protein expression levels of these hub genes in HCC were Validated through the HPA database. Obviously, the protein expression levels of (Fig. 5A) FOXM1, (Fig. 5B) AURKA, (Fig. 5C) CCNA2, (Fig. 5D) MKI67, (Fig. 5E) EZH2, (Fig. 5G)CDK1, (Fig. 5H) CCNB1 and (Fig. 5I) TOP2A were not observed in hepatocytes of normal liver tissues, but medium or high expression levels of these hub genes were detected in HCC tissues (Fig. 5A-E and G-I). The low protein expression levels of CDC6 were detected in normal liver tissues, while medium protein expression levels of CDC6 were detected in HCC tissues (Fig. 5F). Unfortunately,the protein expression levels of CDKN3 were not explored because of pending cancer tissue analysis in HPA database. In brief, these present results showed that the mRNA and protein expression levels of the hub genes were overexpressed in HCC tissues.

Figure 5 Representative immunohistochemistry images of (A) FOXM1, (B) AURKA, (C) CCNA2, (D) MKI67, (E) EZH2, (F) CDC6, (G) CDK1, (H) CCNB1, and (I) TOP2A in HCC and non-cancerous liver tissues derived from the HPA database. HCC, hepatocellular carcinoma; HPA, Human Protein Atlas.

Frequencies of genetic alterations and prognostic values of these hub genes.

The frequencies of genetic alterations of these hub genes in LIHC were identified through the cBioPortal database. $32 \%$ of LIHC cases showed significant alterations in these 10 hub genes (Fig. 6A). The mRNA upregulation was the most important single alteration for these 10 hub genes in 78 cases (22\%) of LIHC. The mRNA expression levels of these 10 hub genes in LIHC were further analyzed. The percentage change in the mRNA expression levels of FOXM1, AURKA, CCNA2, CDKN3, MKI67, EZH2, CDC6, CDK1, CCNB1, and TOP2A in LIHC were 7, 7, 2, 4, 3, 8, 8, 5, 7 and 5\%, respectively (Fig. 6B). Furthermore,the relationship between the changes of these hub genes and LIHC prognosis was established through the cBioportal database. Kaplan-Meier plots were performed to compare OS, and FDS/PFS in LIHC patients with or without alterations in the mRNA expression levels of these hub genes. As showed in Fig. 8C, LIHC cases with altered hub gene showed remarkably worse OS relative to those with unaltered hub gene $(P=$ 5.600e-4). LIHC cases with altered hub gene also showed significantly worse DFS (P = 1.940e-3) compared with those with unaltered hub gene (Fig. 6D).

Figure 6 Alteration frequency and prognosis of the 10 hub genes. (A) The summary of the cancer types was used to calculate alteration frequency of the 10 hub genes in LIHC cases. (B) mRNA expression alterations (RNA Seq V2 RSEM) of the 10 hub genes in LIHC patients. (C) OS and (D) DFS/PFS of LIHC patients with altered (red) and unaltered (blue) mRNA expression of the 10 hub genes. LIHC, liver hepatocellular carcinoma; OS, overall survival; DFS/PFS, disease-free survival/progression-free survival.

\section{Survival analysis of the hub genes in HCC}


To further explore the relationships between the 10 hub genes and HCC, OS and DFS analysis of the 10 hub genes were performed by Kaplan-Meier plotter, and the GEPIA database. As showed in Fig. 7, the high expression levels of FOXM1, AURKA, CCNA2, CDKN3, MKI67, EZH2, CDC6, CDK1, CCNB1, and TOP2A in $\mathrm{LIHC}$ patients were related to poor OS. The unfavorable DFS was also significantly showed in LIHC patients with high expression levels of these 10 hub genes (Fig. 8).

Figure 7 OS of the 10 hub genes overexpressed in patients with liver cancer was analyzed by KaplanMeier plotter. FOXM1, log-rank $P=0.00036$; AURKA, log-rank $P=0.0011 ; C C N A 2$, log-rank $P=0.00018$; $C D K N 3$, log-rank $P=0.0066 ; M K I 67$, log-rank $P=0.00011 ; E Z H 2$, log-rank $P=6.8 e-06 ; C D C 6$, log-rank $P=$ 3.6e-06; CDK1, log-rank $P=1.1 \mathrm{e}-05$; CCNB1, log-rank $P=3.4 \mathrm{E}-05$; and TOP2A, log-rank $P=0.00012$. Data are presented as Log-rank $P$ and the hazard ratio with a $95 \%$ confidence interval. Log-rank $P<0.01$ was regarded as statistically significant. OS, overall survival.

Figure 8 DFS of LIHC patients overexpressed the 10 hub genes were analyzed by the GEPIA online database. Data are presented as log-rank $P$ and the hazard ratio with a $95 \%$ confidence interval. FOXM1, log-rank $P=0.00066$; AURKA, log-rank $P=0.0012$; CCNA2, log-rank $P=0.0037 ; C D K N 3$, log-rank $P=$ 0.0074; MKI67, log-rank $P=4.2 \mathrm{e}-05 ; E Z H 2$, log-rank $P=1 \mathrm{e}-04 ; C D C 6$, log-rank $P=0.0044 ; C D K 1$, log-rank $P$ $=0.00057$; CCNB1, log-rank $P=2.8 \mathrm{E}-06$; and TOP2A, log-rank $P=0.00053$. Log-rank $P<0.01$ was considered statistically significant. DFS, disease-free survival; LIHC, liver hepatocellular carcinoma.

\section{Drug-hub gene interaction}

Using the DGIdb database to explore the drug-gene interactions of the 10 hub genes, 39 drugs for possibly treating HCC were matched and determined (Additional file 1:Table S1). Promising targeted genes of these drugs include AURKB, MKI67, EZH2, TOP2A. The final list only included these drugs which were approved by FDA, and several drugs have been tested in clinical trials. Paclitaxel was considered as a potential drug for cancer therapy due to its inhibition of AURKA and TOP2A. Etoposide, an inhibitor of TOP2A, could inhibit the development of cancer through inducing DNA damaging. Using STITCH database, we constructed downstream networks of AURKA, MKI67, EZH2, and TOP2A to investigate the additional effects caused by inhibitors of these genes. Our models showed that AURKA inhibition might have possible influence on TPX2, microtubule nucleation factor (TPX2), cell division cycle 20 (CDC20), tumor protein p53 (TP53), cell division cycle 25B (CDC25B), baculoviral IAP repeat containing 5 (BIRC5); MKI67 inhibition might have possible influence on AKT serine/threonine kinase 1 (AKT1), cadherin 1 (CDH1); EZH2 inhibition might have possible influence on histone deacetylase 1 (HDAC1), BMI1 protooncogene, polycomb ring finger (BMI1), YY1 transcription factor (YY1), DNA methyltransferase 3 alpha (DNMT3A), DNA methyltransferase 3 beta (DNMT3B), DNA methyltransferase 1(DNMT1), RB binding protein 4(RBBP4), embryonic ectoderm development(EED); TOP2A inhibition might have possible influence on DNA topoisomerase I (TOP1), DNA topoisomerase II beta (TOP2B), ubiquitin C (UBC), proliferating cell nuclear antigen (PCNA), small ubiquitin-like modifer 1 (SUM01), and SUMO2 (Additional file 1: Figures S4-7). So far, few inhibitors of AURKA, MKI67, EZH2, and TOP2A have been tested for HCC therapy. Some of these drugs were even not regarded as anti-cancer drugs (such as levofoxacin and dexrazoxane). These data could provide new insights for targeted therapy in HCC patients. 


\section{Discussion}

In the present study, bioinformatics analysis was performed to identify the potential key genes and biological pathways in HCC. Through comparing the three DEGs profiles of HCC obtained from the GEO database, 54 upregulated DEGs and 143 downregulated DEGs were identified respectively (Fig. 1). Based on the degree of connectivity in PPI network, the 10 hub genes were screened and ranked, including FOXM1, AURKA, CCNA2, CDKN3, MKI67, EZH2, CDC6, CDK1, CCNB1, and TOP2A. These 10 hub genes were functioned as a group, and may play a key role in incidence and prognosis of HCC (Fig. 3A). HCC cases with high expression of the hub genes exhibited significantly worse OS and FDS compared to those with low expression of the hub genes (Figs. 7-8). Additionally, 39 identified drugs provided new insights into the targeted therapies of HCC (Supplemental file: Table S3).

Retinol metabolism, arachidonic acid metabolism, tryptophan metabolism and caffeine metabolism were most markedly enriched for HCC through KEGG pathway enrichment analysis for 197 DGEs. Metabolic alterations clearly characterize HCC tumors [29, 30]. Currently, the rapidly development of metabolomics that allows metabolite analysis in biological fluids is very useful for discovering new biomarkers. Lots of new metabolites have been identified by metabolomics approaches, and some of them could be used as biomarkers in HCC [31].

According to the degree of connectivity, the top 10 genes in PPI network were regarded as hub genes and they were validated in GEPIA database, UCSC Xena browser and HPA database. Many studies reveal that the fork-head box transcription factor FOXM1 is essential for HCC development [32, 33, 34]. Overexpression of FOXM1 has been exhibited to strongly relative to poor prognosis and progression of HCC $[35,36]$. Hepatic progenitor cells of HCC have been identified in the chemical carcinogenesis model, they express cell surface markers CD44 and EpCAM $[32,37]$. Interestingly, deletion of FOXM1 causes disappearance of those cells in the tumor nodules, showing that FOXM1 is critical for the CD44 positive and EpCAM positive HCC cells [32]. The hepatic cancer stem cells in human HCC lines also depend on FOXM1, because deletion of FOXM1 will lead to loss of these cancer stem cells [32]. FOXM1 is a critical downstream factor of many cancer signaling pathways, such as Wnt/ $\beta$-catenin signaling [38]. Moreover, FOXM1 stimulates expression of some multifunctional genes, like c-Myc, Oct4, Sox2 and Nanog [39, 40].

AURKA is a mitotic serine/threonine kinase that regulates cell mitosis, cell division and cell cycle progression [41]. AURKA overexpression has been observed in HCC [42]. And AURKA overexpression has been closely relative to the aggressive tumor characteristics [43], poor prognosis [44] and drug resistance [45] of HCC. AURKA was regulated by c-Myc which contributes to cancer progression in HCC [46]. Alisertib, an inhibitor of AURKA, could inhibit cell viability and induce apoptosis in HCC cells [47]. Wang et al showed genetic variations of AURKA may be a reliable biomarker for the development of HCC [48]. Our study also indicated that increased expression levels of AURKA were relative to unfavorable OS and DFS in HCC patients.

CCNA2 [49] and CCNB1 [50] are two members of the cyclin family, which regulate cell proliferation and apoptosis, and have been close related to cancer progress and patients' survival. CCNA2 [51] and CCNB1 
$[52,53]$ have been identified in various types of tumors. CCNA2 was overexpressed in human HCC tissues [54]. Moreover, it was reported that CCNA2 was relative to a decrease in OS for HCC patients, based on the survival and expression data from TCGA [55]. Liu et al revealed that CCNB1 were highly expressed in HCC tissues compared with normal liver tissues [56]. In addition, the overexpression of CCNB1 was correlated to poor OS and DFS in HCC patients by bioinformatics analysis $[57,58]$. Our study also revealed that HCC patients with a high expression level of CCNA2 or CCNA2 exhibited worse OS and DFS compared to those with a low expression level.

CDKN3 gene is involved in cell mitosis by modulating CDK1/CDK2 dephosphorylation, and its overexpression correlates with unfavorable survival in several cancers [59]. For HCC, CDKN3 not only promotes cell proliferation but also correlates with tumor pathological grade negatively [60]. CDK1, a member of the Ser/Thr protein kinase family, plays an essential role in the control of the eukaryotic cell cycle by modulating the centrosome cycle.CDK1 has been extensively investigated in ovarian cancer and colorectal cancer $[61,62]$. However,little is known about the role of CDK1 in HCC carcinogenesis. A recent study has found that metformin can significantly inhibit the proliferation of HCC cells and effectively reduce the expression of CDK1 [63]. In the present study,the high expression of CDK1 associated with unfavorable OS and DFS in HCC patients.

The maker of proliferation Ki-67'expresses in all phases of the cellular cycle over than $\mathrm{G}_{0}$ phase [64]. MKI67 protein expression in carcinomas has been intensivelyŠinvestigated, and the MKI67-positive cell rate has been shown to be associated with clinical-pathological features and even clinical outcome in various cancers,including HCC [65]. In a study of patients undergoing surgical resection for HCC, higher levels of MKI67 expression in tumor tissue were associated with a higher tumor grade and early tumor recurrence $[66,67]$. Furthermore, staining for MKI67 and P53 are widely used to predict the clinical outcomes of HCC patients after resection and liver transplantation [68].

$\mathrm{EZH} 2$ is a member of the polycomb group (PcG) protein family, which modifies transcription at the epigenetic level by regulating histone and DNA methylation $[69,70]$. Lots of studies have shown that many tumor suppressor genes are suppressed by EZH2 in malignancies and that $\mathrm{EZH} 2$ dysregulation plays a crucial role in carcinogenesis [71, 72]. In our study, the expression of EZH2 was higher in HCC tumor tissue and the high expression of EZH2 was associated with unfavorable OS and DFS in HCC patients.

CDC6 plays a critical role in the initiation of DNA replication. As cells enter the G1 phase, CDC6 binds to the origin recognition complex (ORC) and initiates the assembly of the prereplicative complex (pre-RC) with chromatin licensing and DNA replication factor 1 (CDC1) and mini-chromosome maintenance proteins (MCMs) [73, 74]. Once phosphorylated by cyclin-dependent kinases (CDKs) at the G1/S phase, CDC6 is released from the pre-RC and then DNA is licensed for replication. Growing evidences have suggested that deregulation of CDC6 may contribute to cancer initiation and progression $[75,76]$. Overexpression of the CDC6 protein has been observed in different types of cancer [77]. Our study reveal 
that the expression of $\mathrm{CDC} 6$ was higher in $\mathrm{HCC}$ tumor tissue and the high expression of CDC6 was related with unfavorable OS and DFS in HCC patients.

TOP2A, is a key nuclease that facilitates temporary cleavage and ligation cycle of DNA [78]. In all forms of topoisomerases,TOP2A is predominantly involved in proliferating cells and overexpressed in a variety of cancers (such as breast cancer, urinary bladder cancer, and ovarian carcinoma ) [78]. For HCC, bioinformatics analysis showed that overexpression of TOP2A was common in HCC tumor tissues relative to those in normal liver tissues [79]. Moreover, Wong et al. found that the high expression of TOP2A was correlated with microvascular invasion, advance histological grading, chemotherapy resistance, and poor survival rate [80]. In our study, the expression of TOP2A was higher in HCC tumor tissue compared normal liver tissue, and associated with unfavorable OS and DFS in HCC patients.

A list of 39 drugs with potential therapeutic efficacy against HCC were identified through the DGIdb database. Among the 10 hub genes, the potential gene targeting the drugs are AURKB, MKI67, EZH2, and TOP2A. In Table S2, most of drugs were inhibitors of AURKB, MKI67, EZH2, and TOP2A. However, only a few of these inhibitors have been used for HCC. More studies and clinical trials were needed to identify and explore the effective drugs for HCC. Nevertheless, the present study might push new valuable insights into the individualized and targeted therapy for $\mathrm{HCC}$, and the identified conventional drugs were of potentially new use.

\section{Conclusions}

In summary,the study identifed commonly changed 197 DEGs in HCC through using integrated bioinformatics analysis, including 54 upregulated DEGs and 143 downregulated DEGs. And 10 hub genes(FOXM1, AURKA, CCNA2, CDKN3, MKI67, EZH2, CDC6, CDK1, CCNB1, and TOP2A) might play important roles in HCC. The expression of the hub genes was revealed to be increased in HCC, and the overexpression level predicted poor prognosis. The 10 hub genes might function as novel markers and/or targets for the early HCC detection, prognostic judgment, and targeted therapy of HCC. Additionally, a number of drugs targeting the hub genes were identified, and they could be potentially utilized for treatment of HCC patients. This study provided powerful basis for HCC studies, and further experimental studies were needed.

\section{Abbreviations}

HCC: hepatocellular carcinoma; GEO: Gene Expression Omnibus; DEGs: diferentially expressed genes; GO: gene ontology; KEGG: Kyoto Encyclopedia of Genes and Genomes; PPI: protein-protein interaction; STRING: Search Tool for the Retrieval of Interacting Genes; TCGA: The Cancer Genome Atlas.

\section{Declarations}

Acknowledgements 
We sincerely thank the GEO, Enrichr, STRING, GEPIA, TCGA, HAP, cBioPortal, Kaplan-Meier plotter, DGIdb and STITCH databases for providing their platforms and contributors for their valuable data.

\section{Authors' contributions}

Concept and design: Ping Huang; Analysis and interpretation of the data: Xiaolong Chen; acquisition of data: Xiaolong Chen and Zhixiong Xia; Making diagrams and tables of the article: Xiaolong Chen and Yafeng Wan; drafting of the article: Xiaolong Chen and Zhixiong Xia; critical revision and final approval of the article: Ping Huang.

\section{Funding}

This work was funded by Science and Technology Project of Chongqing Education Commission ,China (Grant No. KJ110317).

\section{Availability of data and materials}

The authors declare that the data supporting this study are available within the article.

\section{Ethics approval and consent to participate}

Not applicable.

\section{Consent for publication}

Not applicable.

\section{Competing interests}

The authors declare that they have no competing interests

\section{References}

1. Jemal A, Bray F, Center MM, Ferlay J, Ward E, Forman D. Global cancer statistics. CA Cancer J Clin. 2011;61:69-90.

2. Llovet JM, Zucman-Rossi J, Pikarsky E, Sangro B, Schwartz M, Sherman M, Gores G. Hepatocellular carcinoma. Nat Rev Dis Primers. 2016;2:16018.

3. Block TM, Mehta AS, Fimmel CJ, Jordan R. Molecular viral oncology of hepatocellular carcinoma. Oncogene. 2003; 22:5093-5107.

4. Si T, Chen Y, Ma D, Gong X, Guan R, Shen B, Peng C. Transarterial chemoembolization prior to liver transplantation for patients with hepatocellular carcinoma: A meta-analysis. J Gastroenterol Hepatol. 2017;32: 1286- 1294. 
5. An P, Xu J, Yu Y, Winkler CA. Host and Viral Genetic Variation in HBV-Related Hepatocellular Carcinoma. Front Genet. 2018; 9:261.

6. Tang R, Liu H, Yuan Y, Xie K, Xu P, Liu X, Wen J. Genetic factors associated with risk of metabolic syndrome and hepatocellular carcinoma. Oncotarget. 2017;8:35403-11.

7. Chen CF, Hsu EC, Lin KT, Tu PH, Chang HW, Lin CH, Chen YJ, Gu DL, Lin CH, Wu JY, Chen YT, Hsu $M T$, Jou YS. Overlapping high-resolution copy number alterations in cancer genomes identified putative cancer genes in hepatocellular carcinoma. Hepatology. 2010,52:1690 - 701.

8. Mžik M, Chmelařová M, John S, Laco J, Slabý O, Kiss I, Bohovicová L, Palička V, Nekvindová J. Aberrant methylation of tumour suppressor genes WT1, GATA5 and PAX5 in hepatocellular carcinoma. Clin Chem Laborat Med. 2016;54:1971-80.

9. Zhen L, Shijie N, Shuijun Z. Tumor PHD2 expression is correlated with clinical features and prognosis of patients with HCC receiving liver resection. Medicine. 2014;93:e179.

10. Lin, S., Hoffmann, K. \& Schemmer P. Treatment of hepatocellular carcinoma: a systematic review. Liver Cancer. 2012; 1:144 - 58.

11. Llovet JM, Ricci S, Mazzaferro V, Hilgard P, Gane E, Blanc JF, de Oliveira AC, Santoro A, Raoul JL, Forner A, Schwartz $M$, Porta $C$, Zeuzem S, et al. Sorafenib in advanced hepatocellular carcinoma. $N$ Engl J Med. 2008;359, 378 - 90.

12. Wang SM, Ooi LL, Hui, KM. Identification and validation of a novel gene signature associated with the recurrence of human hepatocellular carcinoma. Clin Cancer Res. 2007;13:6275-83.

13. Makowska Z, Boldanova T, Adametz D, Quagliata L, Vogt JE, Dill MT, Matter MS, Roth V, Terracciano $L$, Heim MH. Gene expression analysis of biopsy samples reveals critical limitations of transcriptome-based molecular classifications of hepatocellular carcinoma. J Pathol Clin Res. 2016;2:80-92.

14. Schulze K, Imbeaud S, Letouzé E, Alexandrov LB, Calderaro J, Rebouissou S, Couchy G, Meiller C, Shinde J, Soysouvanh F, Calatayud AL, Pinyol R, Pelletier L, Balabaud C, et al. Exome sequencing of hepatocellular carcinomas identifies new mutational signatures and potential therapeutic targets. Nat Genet. 2015;47:505-511.

15. Barrett T, Wilhite SE, Ledoux P, Evangelista C, Kim IF, Tomashevsky M, Marshall KA, Phillippy KH, Sherman PM, Holko M, Yefanov A, Lee H, Zhang N, et al. NCBI GEO: archive for functional genomics data sets-update. Nucleic Acids Res. 2013;41:D991-5.

16. Guo Y, Bao Y, Ma M, Yang W. Identification of Key Candidate Genes and Pathways in Colorectal Cancer by Integrated Bioinformatical Analysis. Int J Mol Sci. 2017;18: 722.

17. Lebrec JJ, Huizinga TW, Toes RE, Houwing-Duistermaat, van Houwelingen HC. Integration of gene ontology pathways with North American Rheumatoid Arthritis Consortium genome-wide association data via linear modeling. BMC Proc. 2009;3:S94.

18. Kuleshov MV, Jones MR, Rouillard AD, Fernandez NF, Duan Q, Wang Z, Koplev S, Jenkins $S L$, Jagodnik KM, Lachmann A, McDermott MG, Monteiro CD, Gundersen GW, et al. Enrichr. A 
comprehensive gene set enrichment analysis web server 2016 update. Nucleic Acids Res. 2016;44:W90-W97.

19. Szklarczyk D, Morris JH, Cook H, Kuhn M, Wyder S, Simonovic M, Santos A, Doncheva NT, Roth A, Bork P, Jensen LJ, von Mering C. The STRING database in 2017: Quality-controlled protein-protein association networks, made broadly accessible. Nucleic Acids Res. 2017;45:D362-D368.

20. Chin CH, Chen SH, Wu HH, Ho CW, Ko MT, Lin CY. CytoHubba: Identifying hub objects and subnetworks from complex interactome. BMC Syst Biol 8, S11 (2014).

21. Tang Z, Li C, Kang B, Gao G, Li C, Zhang Z. GEPIA: A web server for cancer and normal gene expression profiling and interactive analyses. Nucleic Acids Res. 2017;45:W98-W102.

22. Asplund A, Edqvist PH, Schwenk JM, Pontén F. Antibodies for profiling the human proteome-The Human Protein Atlas as a resource for cancer research. Proteomics. 2012;12:2067-2077.

23. Cerami E, Gao J, Dogrusoz U, Gross BE, Sumer SO, Aksoy BA, Jacobsen A, Byrne CJ, Heuer ML, Larsson E, Antipin Y, Reva B, Goldberg AP, et al. The cBio cancer genomics portal: An open platform for exploring multidimensional cancer genomics data. Cancer Discov. 2012;2:401-404.

24. Menyhárt O, Nagy Á, Györffy B. Determining consistent prognostic biomarkers of overall survival and vascular invasion in hepatocellular carcinoma. $R$ Soc Open Sci. 2018;5:181006.

25. Cotto KC, Wagner AH, Feng YY, Kiwala S, Coffman AC, Spies G, Wollam A, Spies NC, Griffith OL, Griffith M. DGIdb 3.0: a redesign and expansion of the drug-gene interaction database. Nucleic Acids Res. 2018;46:D1068-73.

26. Kirk J, Shah N, Noll B, Stevens CB, Lawler M, Mougeot FB, Mougeot JC. Text mining-based in silico drug discovery in oral mucositis caused by high-dose cancer therapy. Support Care Cancer. 2018;26:2695 - 705.

27. Szklarczyk D, Santos A, von Mering C, Jensen LJ, Bork P, Kuhn M. STITCH 5: augmenting proteinchemical interaction networks with tissue and afnity data. Nucleic Acids Res. 2016;44:D380-4.

28. Pathan M, Keerthikumar S, Ang CS, Gangoda L, Quek CY, Williamson NA, Mouradov D, Sieber OM, Simpson RJ, Salim A, Bacic A, Hill AF, Stroud DA. FunRich: an open access standalone functional enrichment and interaction network analysis tool. Proteomics. 2015;15:2597 - 601.

29. Shang RZ, Qu SB, Wang DS. Reprogramming of glucose metabolism in hepatocellular carcinoma: progressand prospects. World Journal of Gastroenterology. 2016;22:9933-9943.

30. Kitamura K, Hatano E, Higashi T, Narita M, Seo S, Nakamoto Y, Yamanaka K, Nagata H, Taura K, Yasuchika K, Nitta T, Uemoto S. Proliferative activity in hepatocellular carcinoma is closely correlated with glucose metabolism but not angiogenesis. Journal of Hepatology. 2011;55:846-857.

31. Kornberg A, Freesmeyer M, Bärthel E, Jandt K, Katenkamp K, Steenbeck J, Sappler A, Habrecht O, Gottschild D, Settmacher U. 18F-FDG uptake of hepatocellular carcinoma on PET predicts microvascular tumor invasion in liver transplant patients. American Journal of Transplantation. 2009;9:592-600.

32. Kopanja D, Pandey A, Kiefer M, Wang Z, Chandan N, Carr JR, Franks R, Yu DY, Guzman G, Maker A, Raychaudhuri P. Essential roles of FoxM1 in Ras-induced liver cancer progression and in cancer cells 
with stem cell features. J Hepatol. 2015;63:429 - 36.

33. Kalinichenko VV, Major ML, Wang X, Petrovic V, Kuechle J, Yoder HM, Dennewitz MB, Shin B, Datta A, Raychaudhuri $P$, Costa RH. Foxm1b transcription factor is essential for development of hepatocellular carcinomas and is negatively regulated by the p19ARF tumor suppressor. Genes Dev. 2004; $18: 830-50$.

34. Park HJ, Gusarova G, Wang Z, Carr JR, Li J, Kim KH, Qiu J, Park YD, Williamson PR, Hay N, Tyner AL, Lau LF, Costa RH, et al. Deregulation of FoxM1b leads to tumour metastasis. EMBO Mol Med. 2017;3:21-34.

35. Sun H, Teng M, Liu J, Jin D, Wu J, Yan D, Fan J, Qin X, Tang H, Peng Z. FOXM1 expression predicts the prognosis in hepatocellular carcinoma patients after orthotopic liver transplantation combined with the Milan criteria. Cancer Lett. 2011;306:214 - 22.

36. Sun HC, Li M, Lu JL, Yan DW, Zhou CZ, Fan JW, Qin XB, Tang HM, Peng ZH. Overexpression of Forkhead box $M 1$ protein associates with aggressive tumor features and poor prognosis of hepatocellular carcinoma. Oncol Rep. 2011;25:1533-9.

37. He G, Dhar D, Nakagawa H, Font-Burgada J, Ogata H, Jiang Y, Shalapour S, Seki E, Yost SE, Jepsen K, Frazer KA, Harismendy $O$, Hatziapostolou M, et al. Identification of liver cancer progenitors whose malignant progression depends on autocrine IL-6 signaling. Cell 155, 384 - 96 (2013).

38. Gong A, Huang S. FoxM1 and Wnt/beta-catenin signaling in glioma stem cells. Cancer Res. 2012; 72:5658-62.

39. Xie Z, Tan G, Ding M, Dong D, Chen T, Meng X, Huang X, Tan Y. Foxm1 transcription factor is required for maintenance of pluripotency of P19 embryonal carcinoma cells. Nucleic Acids Res. 2010;38:8027-38.

40. Bella L, Zona S, Nestal de Moraes G, Lam EW. FOXM1: A key oncofoetal transcription factor in health and disease. Semin Cancer Biol. 2014;29:32 - 9.

41. Vader G, Lens SM. The Aurora kinase family in cell division and cancer. Biochim Biophys Acta 1786, 60-72(2008).

42. Simon EP, Freije CA, Farber BA, Lalazar G, Darcy DG, Honeyman JN, Chiaroni-Clarke R, Dill BD, Molina $H$, Bhanot UK, La Quaglia MP, Rosenberg BR, Simon SM. Transcriptomic characterization of fibrolamellar hepatocellular carcinoma. Proc Natl Acad Sci USA. 2005; 112:E5916-E5925.

43. Jeng YM, Peng SY, Lin CY, Hsu HC. Overexpression and amplification of Aurora-A in hepatocellular carcinoma. Clin Cancer Res. 2004; 10:2065-2071.

44. Chen $C$, Song G, Xiang J, Zhang H, Zhao S, Zhan Y. AURKA promotes cancer metastasis by regulating epithelial-mesenchymal transition and cancer stem cell properties in hepatocellular carcinoma. Biochem Biophys Res Commun. 2007;486:514-520.

45. Zhang K, Chen J, Chen D, Huang J, Feng B, Han S, Chen Y, Song H, De W, Zhu Z, Wang R, Chen L. Aurora-A promotes chemoresistance in hepatocelluar carcinoma by targeting NF-kappaB/microRNA21/PTEN signaling pathway. Oncotarget. 2014;5:12916-12935. 
46. Lu L, Han H, Tian Y, Li W, Zhang J, Feng M, Li Y. Aurora kinase A mediates c-Myc's oncogenic effects in hepatocellular carcinoma. Mol Carcinog. 2015;54:1467-1479.

47. Li X, Xu W, Kang W, Wong SH, Wang M, Zhou Y, Fang X, Zhang X, Yang H, Wong CH, To KF, Chan SL, Chan MTV. Genomic analysis of liver cancer unveils novel driver genes and distinct prognostic features. Theranostics. 2018;8:1740-1751.

48. Wang B, Hsu CJ, Chou CH, Lee HL, Chiang WL, Su CM, Tsai HC, Yang SF, Tang CH. Variations in the AURKA gene: Biomarkers for the development and progression of hepatocellular carcinoma. Int $J$ Med Sci. 2018;15:170-175.

49. Volm M, Koomägi R, Mattern J, Stammler G. Cyclin A is associated with an unfavourable outcome in patients with non-small-cell lung carcinomas. Br J Cancer. 1997;75:1774-1778.

50. Ding K, Li W, Zou Z, Zou X, Wang C. CCNB1 is a prognostic biomarker for ER + breast cancer. Med Hypotheses. 2014;83:359-364.

51. Handa K, Yamakawa M, Takeda H, Kimura S, Takahashi T. Expression of cell cycle markers in colorectal carcinoma: Superiority of cyclin A as an indicator of poor prognosis. Int J Cancer. 1990;84:225-233.

52. Wang A, Yoshimi N, Ino N, Tanaka T, Mori H. Overexpression of cyclin B1 in human colorectal cancers. J Cancer Res Clin Oncol. 1997;123:124-127.

53. Soria JC, Jang SJ, Khuri FR, Hassan K, Liu D, Hong WK, Mao L. Overexpression of cyclin B1 in earlystage non-small cell lung cancer and its clinical implication. Cancer Res. 2000;60:4000-4004.

54. Andrisani OM, Studach L, Merle P. Gene signatures in hepatocellular carcinoma (HCC). Semin Cancer Biol. 2011;21:4-9.

55. Chen QF, Xia JG, Li W, Shen LJ, Huang T, Wu P. Examining the key genes and pathways in hepatocellular carcinoma development from hepatitis B virus-positive cirrhosis. Mol Med Rep. 2018; 18:4940-4950.

56. Liu S, Yao X, Zhang D, Sheng J, Wen X, Wang Q, Chen G, Li Z, Du Z, Zhang X. Analysis of transcription factor-related regulatory networks based on bioinformatics analysis and validation in hepatocellular carcinoma. Biomed Res Int. 2018,2018: 1431396.

57. Gao X, Wang X, Zhang S. Bioinformatics identification of crucial genes and pathways associated with hepatocellular carcinoma. Biosci Rep. 2018;38:BSR20181441.

58. Zhuang L, Yang Z, Meng Z. Upregulation of BUB1B, CCNB1, CDC7, CDC20, and MCM3 in tumor tissues predicted worse overall survival and disease-free survival in hepatocellular carcinoma patients. Biomed Res Int. 2018;2018: 7897346.

59. Cress $W D, Y u P, W u$ J. Expression and alternative splicing of the cyclin-dependent kinase inhibitor-3 gene in human cancer. Int. J. Biochem. Cell Biol. 2017;91:98-101.

60. Dai W, Miao H, Fang S, Fang T, Chen N, Li M. CDKN3 expression is negatively associated with pathological tumor stage and CDKN3 inhibition promotes cell survival in hepatocellular carcinoma. Mol. Med. Rep. 2016;14:1509-1514. 
61. Yang W, Cho H, Shin HY, Chung JY, Kang ES, Lee EJ, Kim JH. Accumulation of cytoplasmic Cdk1 is associated with cancer growth and survival rate in epithelial ovarian cancer. Oncotarget. 2016; 7:49481-97.

62. Zhang R, Shi H, Ren F, Zhang M, Ji P, Wang W, Liu C. The aberrant upstream pathway regulations of CDK1 protein were implicated in the proliferation and apoptosis of ovarian cancer cells. J Ovarian Res. 2017;10:60.

63. Zhou J, Han S, Qian W, Gu Y, Li X, Yang K. Metformin induces miR-378 to downregulate the CDK1, leading to suppression of cell proliferation in hepatocellular carcinoma. OncoTargets and Therapy. 2018;11:4451-4459.

64. Gerdes J, Lemke H, Baisch H, Wacker HH, Schwab U, Stein H. Cell cycle analysis of a cell proliferation-associated human nuclear antigen defined by the monoclonal antibody Ki-67. J Immunol. 1984; 133: 1710-5.

65. Luo Y, Ren F, Liu Y, Shi Z, Tan Z, Xiong H, Dang Y, Chen G. Clinicopathological and prognostic significance of high Ki-67 labeling index in hepatocellular carcinoma patients: a meta-analysis. Int J Clin Exp Med. 2105;8:10235-47.

66. D'Errico A, Grigioni WF, Fiorentino M, Baccarini P, Grazi GL, Mancini AM. Overexpression of p53 protein and Ki67 proliferative index in hepatocellular carcinoma: an immunohistochemical study on 109 Italian patients. Pathol Int. 1994;44:682-7.

67. Nakanishi K, Sakamoto M, Yamasaki S, Todo S, Hirohashi S. Akt phosphorylation is a risk factor for early disease recurrence and poor prognosis in hepatocellular carcinoma. Cancer. 2005;103:307 12.

68. Guzman G, Alagiozian-Angelova V, Layden-Almer JE, Layden TJ, Testa G, Benedetti E, Kajdacsy-Balla A, Cotler SJ. p53, Ki-67, and serum alpha feto-protein as predictors of hepatocellular carcinoma recurrence in liver transplant patients. Mod Pathol. 2005; 18:1498 - 503.

69. Gao SB, Zheng QF, Xu B, Pan CB, Li KL, Zhao Y, Zheng QL, Lin X, Xue LX, Jin GH. EZH2 represses target genes through H3K27-dependent and H3K27-independent mechanisms in hepatocellular carcinoma. Mol Cancer Res. 2014;12:1388-97.

70. Kondo Y, Shen L, Cheng AS, Ahmed S, Boumber Y, Charo C, Yamochi T, Urano T, Furukawa K, KwabiAddo B, Gold DL, Sekido Y, Huang TH, Issa JP. Gene silencing in cancer by histone H3 lysine 27 trimethylation independent of promoter DNA methylation. Nat Genet. 2008;40:741 - 50.

71. Xu B, Konze KD, Jin J, Wang GG. Targeting EZH2 and PRC2 dependence as novel anticancer therapy. Exp Hematol. 2015;43:698-712.

72. Au SL, Wong CC, Lee JM, Fan DN, Tsang FH, Ng IO, Wong CM. Enhancer of zeste homolog 2 epigenetically silences multiple tumor suppressor microRNAs to promote liver cancer metastasis. Hepatology. 2012;56:622 - 31.

73. Randell JC, Bowers JL, Rodriguez HK, Bell SP. Sequential ATP hydrolysis by Cdc6 and ORC directs loading of the Mcm2-7 helicase. Mol. Cell. 2006;21:29-39. 
74. Speck C, Stillman B. Cdc6 ATPase activity regulates ORC-Cdc6 stability and the selection of specific DNA sequences as origins of DNA replication. J. Biol. Chem. 2007;282:11705-11714.

75. Liontos M, Koutsami M, Sideridou M, Evangelou K, Kletsas D, Levy B, Kotsinas A, Nahum O, Zoumpourlis V, Kouloukoussa M, Lygerou Z, Taraviras S, Kittas C, et al. Deregulated overexpression of hCdt1 and hCdc6 promotes malignant behavior. Cancer Res. 2007;67:10899-10909.

76. Borlado LR, Mendez J. CDC6: from DNA replication to cell cycle checkpoints and oncogenesis. Carcinogenesis. 2008; 29:237-243.

77. Semple JW, Duncker BP. ORC-associated replication factors as biomarkers for cancer. Biotechnol. Adv. 2004;22:621-631.

78. Ali Y, Abd Hamid S. Human topoisomerase II alpha as a prognostic biomarker in cancer chemotherapy. Tumour Biol. 2016;37:47-55.

79. Zhou L, Du Y, Kong L, Zhang X, Chen Q. Identification of molecular target genes and key pathways in hepatocellular carcinoma by bioinformatics analysis. Oncol. Targets Ther. 2018;11:1861-1869.

80. Wong N, Yeo W, Wong WL, Wong NL, Chan KY, Mo FK, Koh J, Chan SL, Chan AT, Lai PB, Ching AK, Tong $\mathrm{JH}, \mathrm{Ng} H K$, et al. TOP2A overexpression in hepatocellular carcinoma correlates with early age onset, shorter patients survival and chemoresistance. Int. J. Cancer. 2009;124:644-652.

\section{Supplemental Information}

Additional file 1. Figure S1: The heatmap of 197 DEGs using data profile GSE64041 as a reference. Figure S2: PPI network was constructed by all the 197 DEGs using STRING database. Figure S3: Hierarchical clustering analysis of the hub genes in HCC $(n=469)$ and normal liver tissue $(n=50)$ was conducted using the UCSC Xena browser. Figure S4: Drug-hub genes network of AURKA. Figure S5: Drug-hub genes network of MKI67. Figure S6: Drug-hub genes network of EZH2. Figure S7: Drug-hub genes network of TOP2A. Table S1: Candidate drugs targeting hub genes.

\section{Figures}



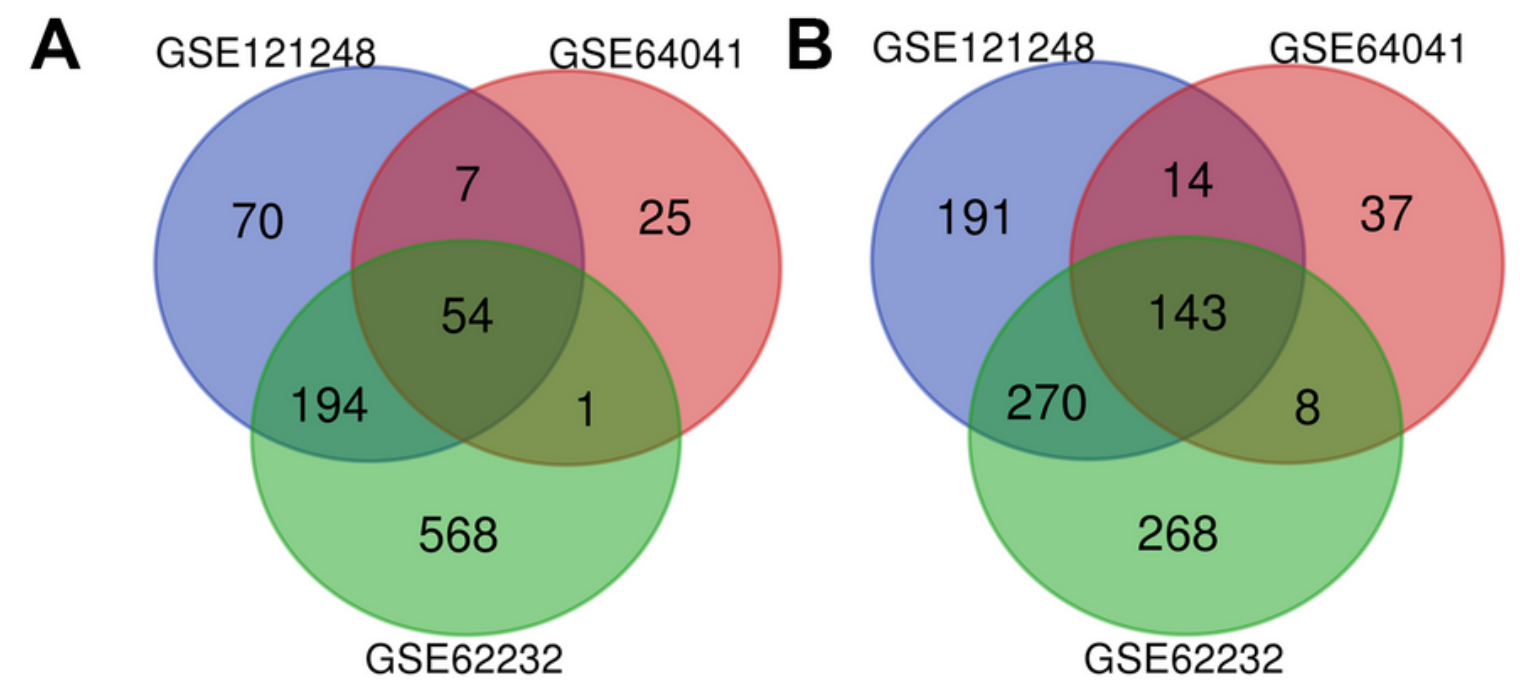

C

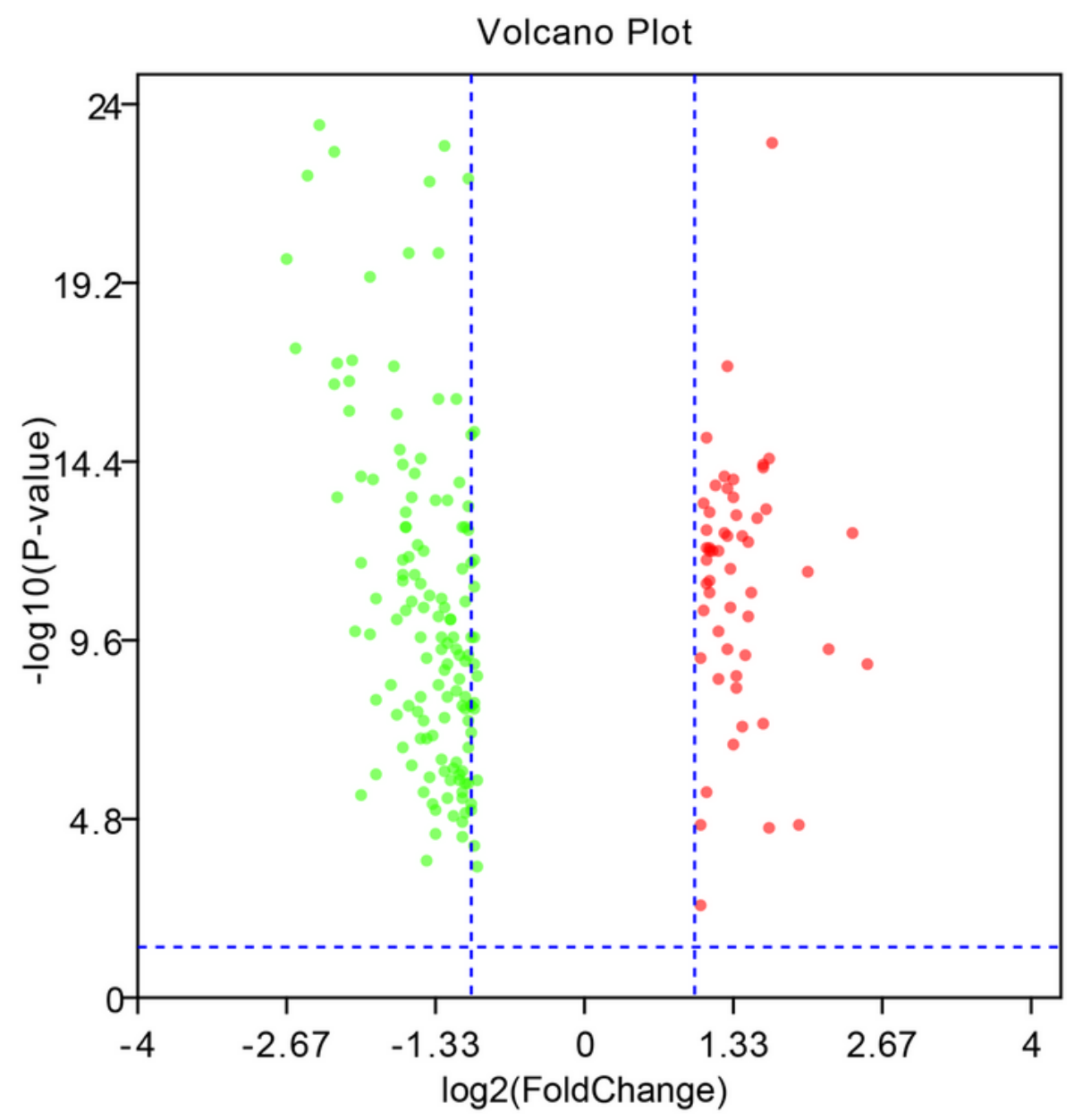

\section{Figure 1}

Identification of common DEGs from GSE121248, GSE64041, and GSE62232 datasets. Venn diagram of (A) upregulated and (B) downregulated DEGs based on the three GEO datasets. (C) Volcano plot of the 197 DEGs. Red, upregulation; green, downregulation. The intersecting areas represent the commonly altered DEGs. The t-test was used to analyze DEGs, with the cut-off criteria of $\| \log F C \mid>1.0$ and adj. $P<0.05$. DEGs, differentially expressed genes; GEO, Gene Expression Omnibus; logFC, log-fold change. 

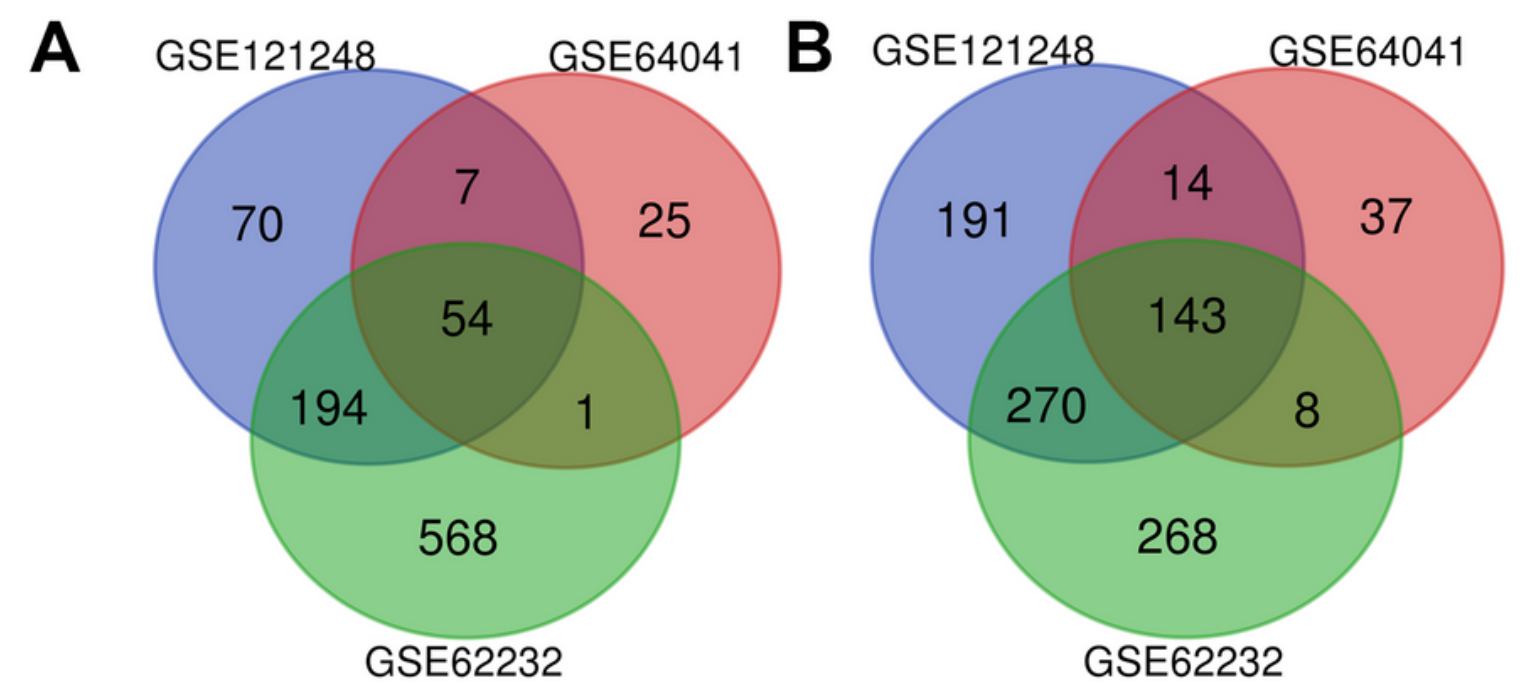

C

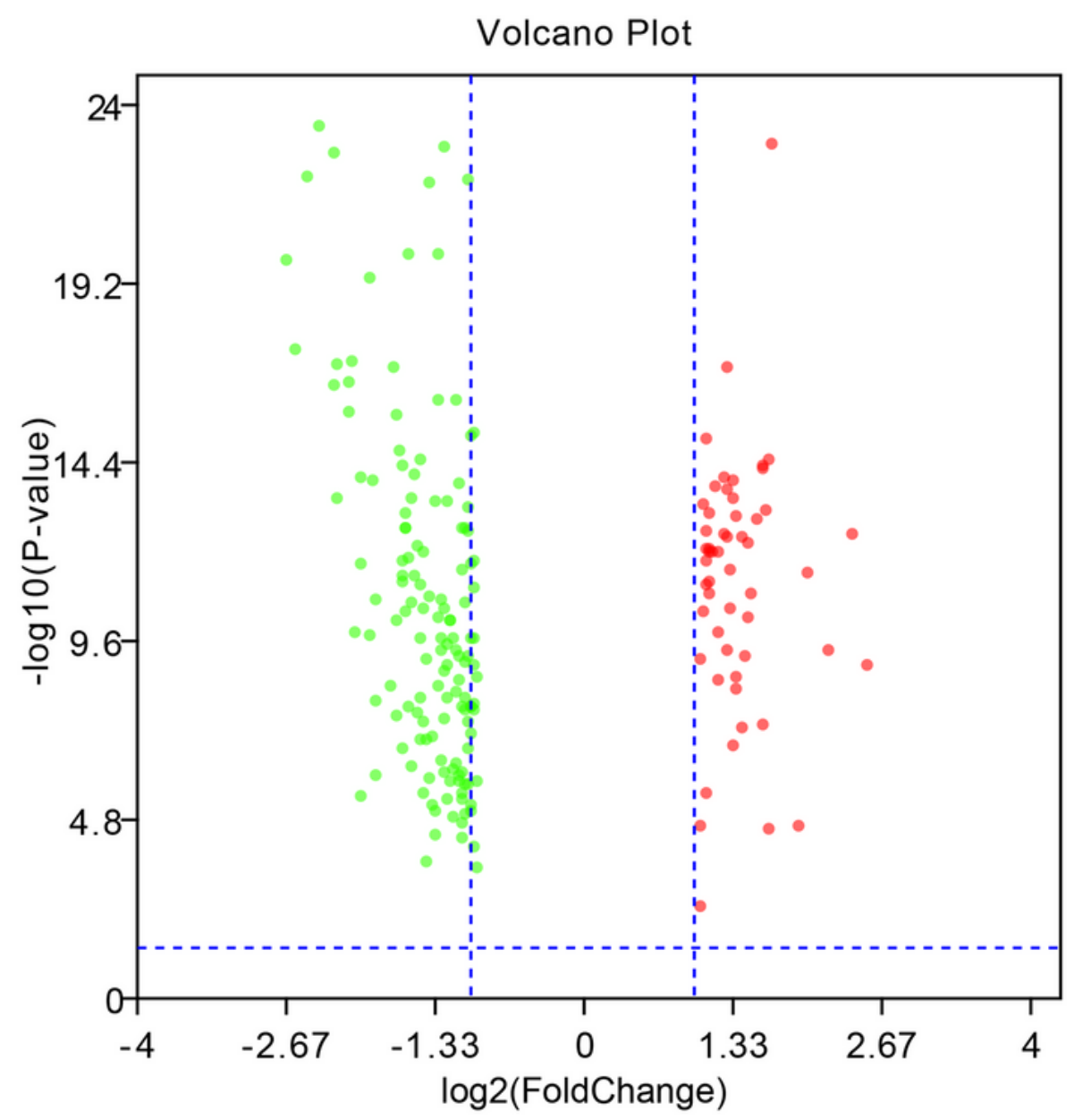

\section{Figure 1}

Identification of common DEGs from GSE121248, GSE64041, and GSE62232 datasets. Venn diagram of (A) upregulated and (B) downregulated DEGs based on the three GEO datasets. (C) Volcano plot of the 197 DEGs. Red, upregulation; green, downregulation. The intersecting areas represent the commonly altered DEGs. The t-test was used to analyze DEGs, with the cut-off criteria of $\|$ ogFC| $>1.0$ and adj. $P<0.05$. DEGs, differentially expressed genes; GEO, Gene Expression Omnibus; logFC, log-fold change. 


\section{epoxygenase P450 pathway (CO:0019373)}

steroid metabolic process (CO:0008202)

kynurenine metabolic process (CO:0070189

arachidonic acid metabolic process (CO:0019369)

omega-hydroxylase P450 parthway (CO:0097267)

indolalkylamine catabolic process (CO:0046218)

aromatic amino acid family catabolic process (CO:0009074)

tryptophan m tabolic process (CO:0006568)

tryptophan carabolic process (GO:0006569)

cell chemotaxis (CO:0060326)

B

Cellular Component

condensed nuclear chromosome, centromeric region (CO:0000780)

spindle (CO:0005819)

integral component of plasma membrane (CO:0005887)

condensed nuclear chromosome kinetochore (CO:0000778)

mitotic spindle (CO:0072686)

spindle pole (CO:0000922)

condensed chromosome kinetochore (CO:0000777)

spindle microtubule (CO:0005876)

membrane attack complex (CO:0005579)

condensed chromosome, centromeric region (CO:0000779)

C

Molecular Function

oxidoreductase activity, acting on the $\mathrm{CH}-\mathrm{OH}$ group of donors, NAD or NADP as acceptor (CO:0016616)

steroid hydroxylase activity (CO:0008395)

oxidoreductase activity, acting on paired donors, with incorporation or reduction of molecular oxygen, NAD(P)H as one d

heme binding (CO:0020037)

arachidonic acid epoxygenase activity (CO:0008392)

arachidonic acid monooxygenase activity (CO:0008391)

peptidase inhibitor activity (CO:0030414)

oxidoreductase activity, acting on paired donors, with incorporation or reduction of molecular oxygen, reduced flavin or

histone kinase activity (CO:0035173)

metal ion binding (GO:0046872)

D

KEGG

Retinol metabolism
Arachidonic acid metabolism
Tryptophan metabolism
Caffeine metabolism
Drug metabolism
Chemical carcinogenesis
Linoleic acid metabolism
Progesterone-mediated oocyte maturation
Primary bile acid biosynthesis
Clycine, serine and threonine metabolism

Figure 2

GO annotation and KEGG pathway enrichment analysis of 197 DEGs. The top 10 enriched GO (A) BP, (B) CC, (C) MF terms and (D) KEGG pathways. GO, Gene Ontology; KEGG, Kyoto Encyclopedia of Genes and Genomes; DEGs, differentially expressed genes; BP, biological process; CC, cellular component; MF, molecular function. 


\section{epoxygenase P450 pathway (CO:0019373)}

steroid metabolic process (CO:0008202)

kynurenine metabolic process (CO:0070189)

arachidonic acid metabolic process (CO:0019369)

omega-hydroxylase P450 patthway (CO:0097267)

indolalkylamine catabolic process (CO:0046218)

aromatic amino acid family catabolic process (CO:0009074)

tryptophan mftabolic process (CO:0006568)

tryptophan carabolic process (GO:0006569)

cell chemotaxis (CO:0060326)

B

Cellular Component

condensed nuclear chromosome, centromeric region (CO:0000780)

spindle (CO:0005819)

integral component of plasma membrane (CO:0005887)

condensed nuclear chromosome kinetochore (CO:0000778)

mitotic spindle (CO:0072686)

spindle pole (CO:0000922)

condensed chromosome kinetochore (CO:0000777)

spindle microtubule (CO:0005876)

membrane attack complex (CO:0005579)

condensed chromosome, centromeric region (CO:0000779)

C

Molecular Function

oxidoreductase activity, acting on the $\mathrm{CH}-\mathrm{OH}$ group of donors, NAD or NADP as acceptor (CO:0016616)

steroid hydroxylase activity (CO:0008395)

oxidoreductase activity, acting on paired donors, with incorporation or reduction of molecular oxygen, NAD(P)H as one d

heme binding (CO:0020037)

arachidonic acid epoxygenase activity (CO:0008392)

arachidonic acid monooxygenase activity (CO:0008391)

peptidase inhibitor activity (CO:0030414)

oxidoreductase activity, acting on paired donors, with incorporation or reduction of molecular oxygen, reduced flavin or

histone kinase activity (CO:0035173)

metal ion binding (CO:0046872)

D

KEGG

Retinol metabolism
Arachidonic acid metabolism
Tryptophan metabolism
Caffeine metabolism
Drug metabolism
Chemical carcinogenesis
Linoleic acid metabolism
Progesterone-mediated oocyte maturation
Primary bile acid biosynthesis
Clycine, sefine and threonine metabolism

Figure 2

GO annotation and KEGG pathway enrichment analysis of 197 DEGs. The top 10 enriched GO (A) BP, (B) CC, (C) MF terms and (D) KEGG pathways. GO, Gene Ontology; KEGG, Kyoto Encyclopedia of Genes and Genomes; DEGs, differentially expressed genes; BP, biological process; CC, cellular component; $\mathrm{MF}$, molecular function. 
A

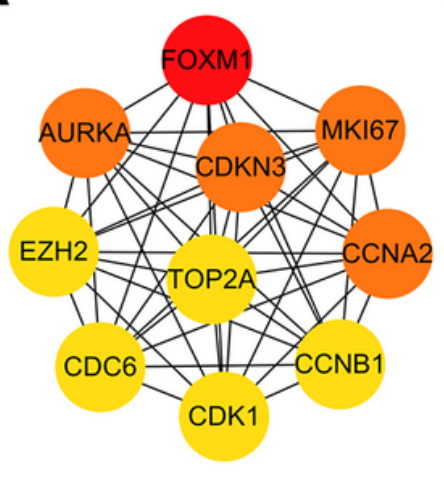

C Progesterone-mediated oocyte maturation

Cell cycle

Cellular senescence

Oocyte meiosis

p53 signaling pathway

Viral carcinogenesis

Human immunodeficiency virus 1 infection

Lysine degradation

Gap junction

AMPK signaling pathway

B

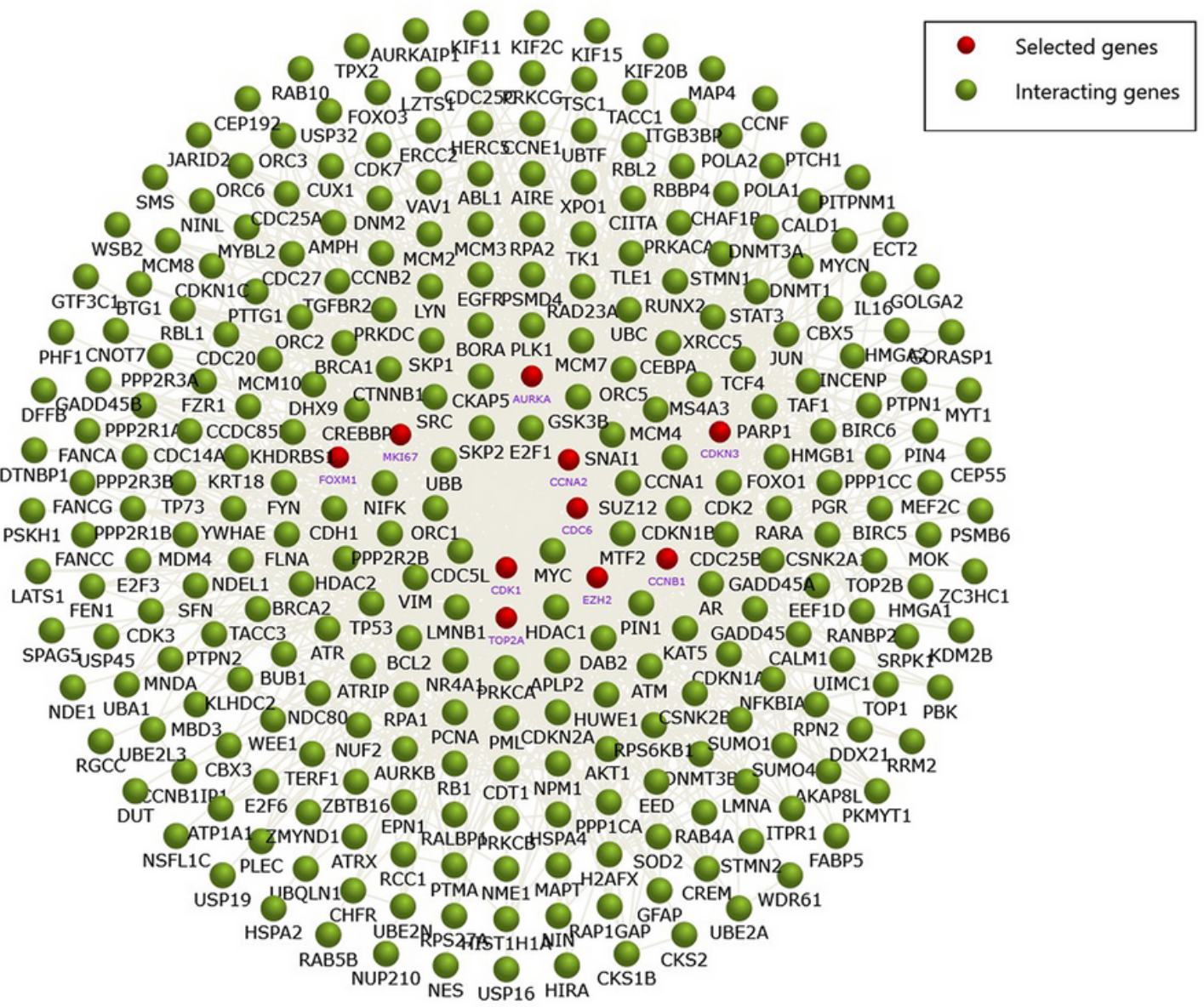

\section{Figure 3}

Interaction network and KEGG analysis of the hub genes. (A) The top 10 hub genes in the PPI network were screened by Cytoscape (v3.6.1) plugin cytoHubba. The 10 hub genes are displayed from red (high degree value) to yellow (low degree value). (B)The PPI network of the 10 hub genes and their related genes, created by the FunRich software. (C) KEGG pathway enrichment analysis of the 10 hub genes. PPI, protein-protein interaction ; STRING, search tool for the retrieval of interacting genes; KEGG, Kyoto encyclopedia of genes and genomes. 
A

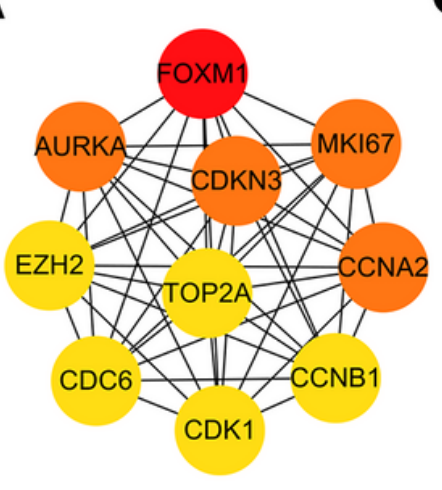

C

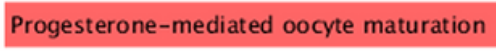

\section{Cell cycle}

Cellular senescence

Oocyte meiosis

\section{p53 signaling pathway}

Viral carcinogenesis

Human immunodeficiency virus 1 infection

Lysine degradation

Gap junction

AMPK signaling pathway

B

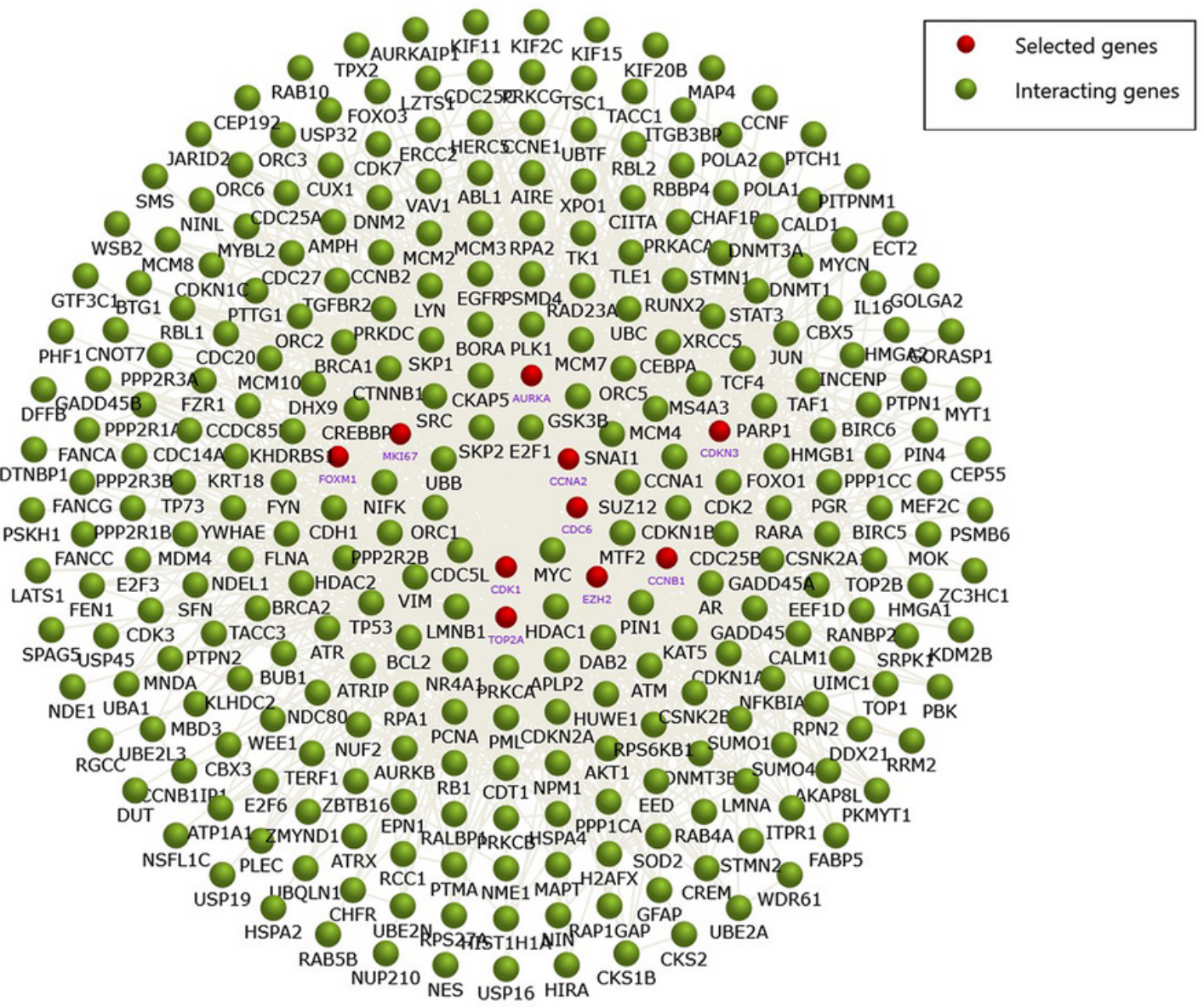

\section{Figure 3}

Interaction network and KEGG analysis of the hub genes. (A) The top 10 hub genes in the PPI network were screened by Cytoscape (v3.6.1) plugin cytoHubba. The 10 hub genes are displayed from red (high degree value) to yellow (low degree value). (B)The PPI network of the 10 hub genes and their related genes, created by the FunRich software. (C) KEGG pathway enrichment analysis of the 10 hub genes. PPI, protein-protein interaction ; STRING, search tool for the retrieval of interacting genes; KEGG, Kyoto encyclopedia of genes and genomes. 

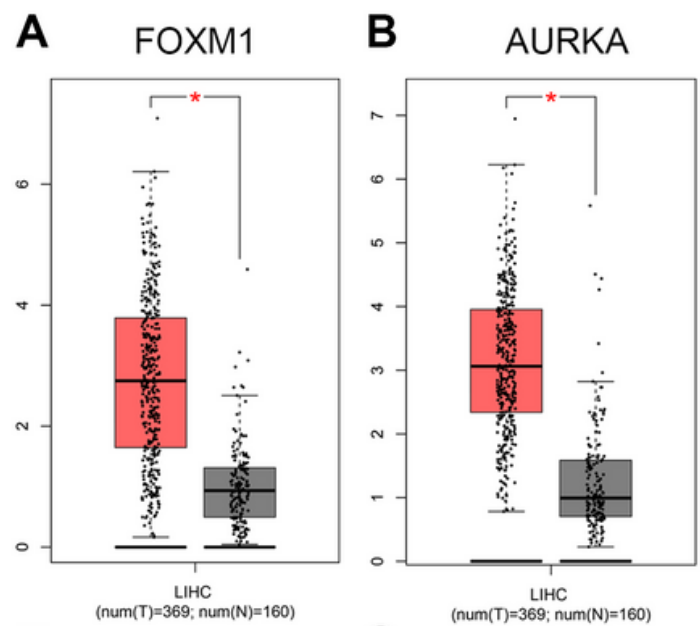

C CCNA2

D $\quad$ CDKN3

E $\quad$ MKI67

$\mathrm{F}$

G
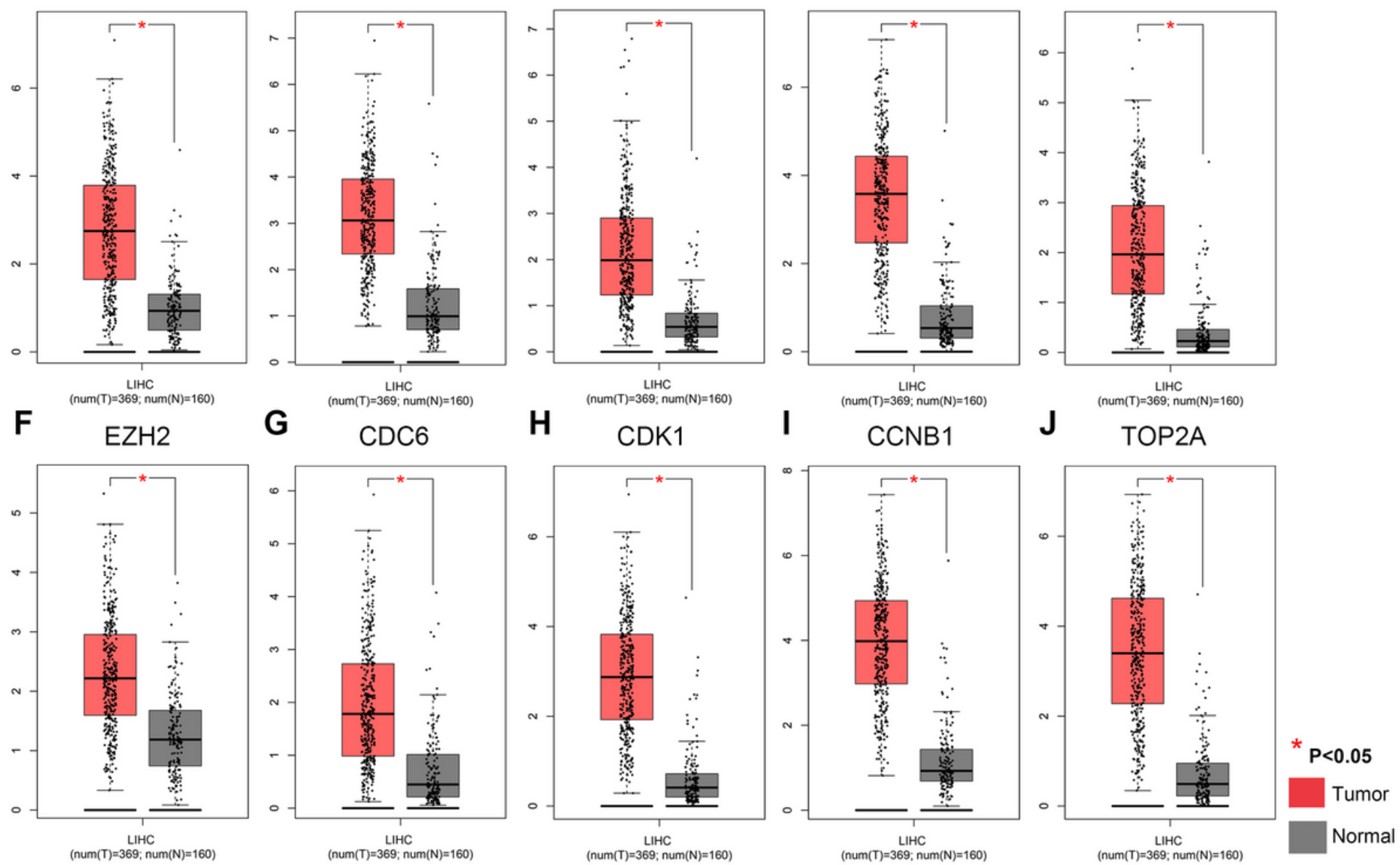

Figure 4

Validation of the mRNA expression levels of (A) FOXM1, (B) AURKA, (C) CCNA2, (D) CCKN3, (E) MKI67, (F) EZH2, (G) CDC6, (H) CDK1, (I) CCNB1, and (J) TOP2A in LIHC tissues and normal liver tissues using GEPIA database. These ten box plots are based on 369 LIHC samples (marked in red) and 160 normal samples (marked in gray). ${ }^{*}<0.01$ was considered statistically significant. LIHC, liver hepatocellular carcinoma. 


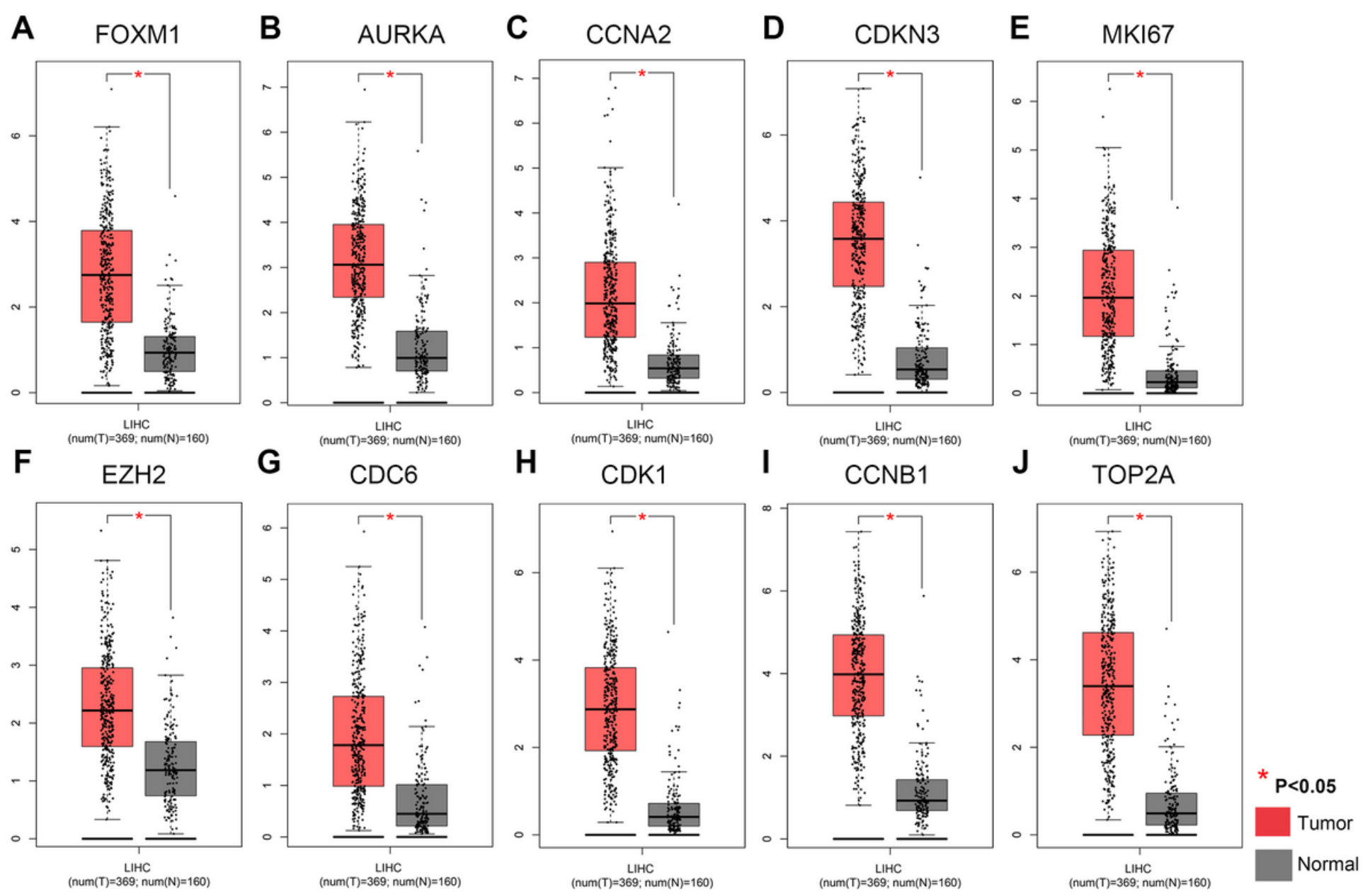

Figure 4

Validation of the mRNA expression levels of (A) FOXM1, (B) AURKA, (C) CCNA2, (D) CCKN3, (E) MKI67, (F) EZH2, (G) CDC6, (H) CDK1, (I) CCNB1, and (J) TOP2A in LIHC tissues and normal liver tissues using GEPIA database. These ten box plots are based on $369 \mathrm{LIHC}$ samples (marked in red) and 160 normal samples (marked in gray). ${ }^{*}<0.01$ was considered statistically significant. LIHC, liver hepatocellular carcinoma. 


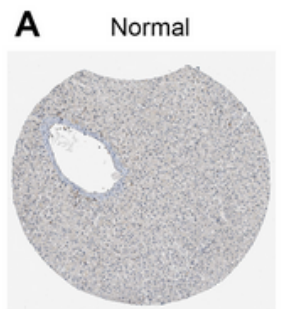

Staining: Not detected
Tumor

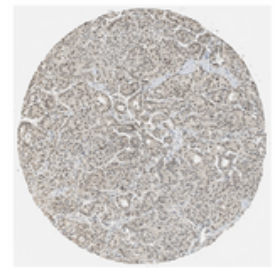

Staining: Medium

C

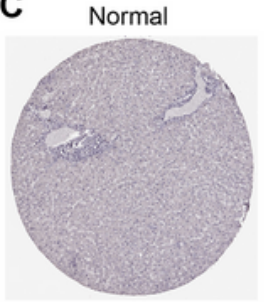

Staining: Not detected
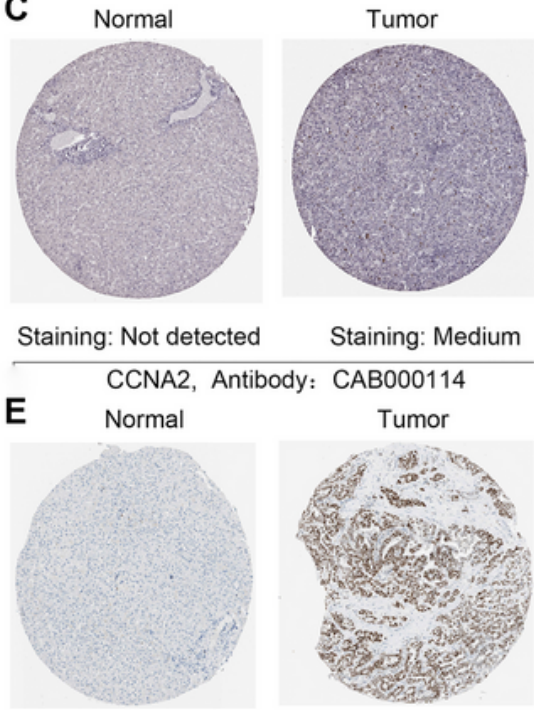

Staining: Medium
Staining: Not detected

G Normal

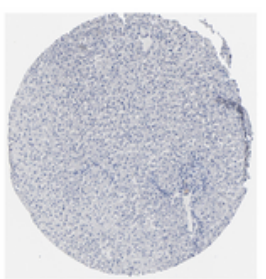

Staining: Not detected

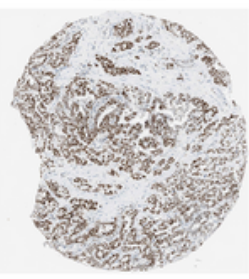

Staining: High

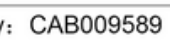

\begin{abstract}
y: CAB009589
\end{abstract}

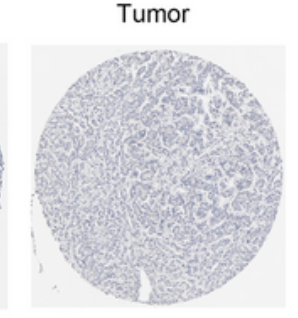

Staining: Medium

CDK1, Antibody: CAB003799

I Normal

Tumor

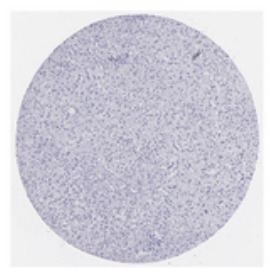

Staining: Not detected

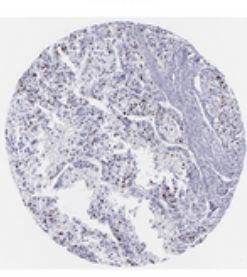

Staining: Medium TOP2A, Antibody: CAB002448
B

Normal
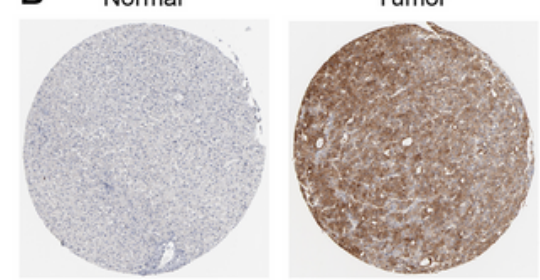

Staining: Not detected

Staining: Medium

AURKA, Antibody: HPA002636

D
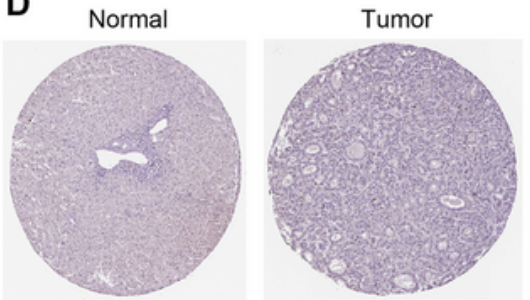

Staining: Not detected

Staining: Medium

MKI67, Antibody: CAB000058

F Normal

Tumor
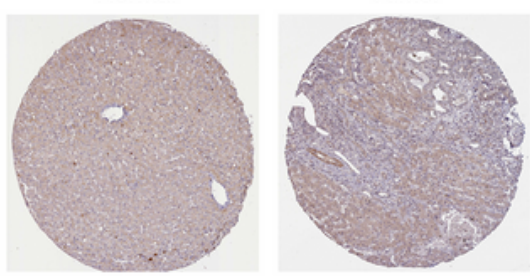

Staining: Low

Staining: Medium

CDC6, Antibody: HPA050114

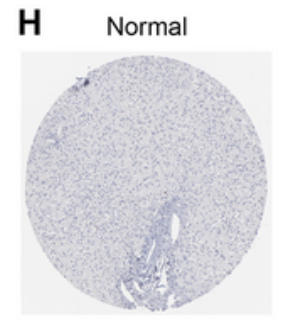

Tumor

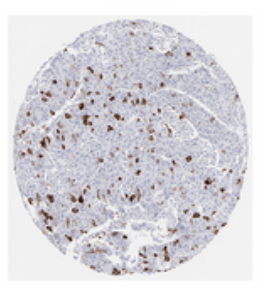

Staining: Not detected

Staining: Medium

CCNB1, Antibody: CAB003804

\section{Figure 5}

Representative immunohistochemistry images of (A) FOXM1, (B) AURKA, (C) CCNA2, (D) MKI67, (E) EZH2, (F) CDC6, (G) CDK1, (H) CCNB1, and (I) TOP2A in HCC and non-cancerous liver tissues derived from the HPA database. HCC, hepatocellular carcinoma; HPA, Human Protein Atlas. 


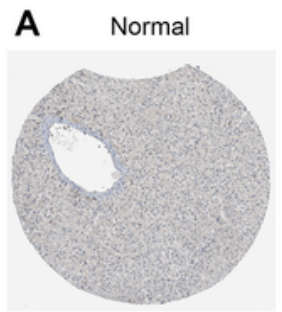

Staining: Not detected
Tumor

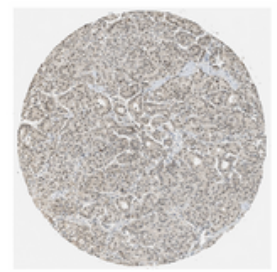

Staining: Medium

C

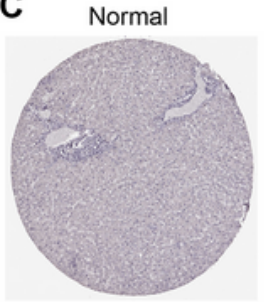

Staining: Not detected
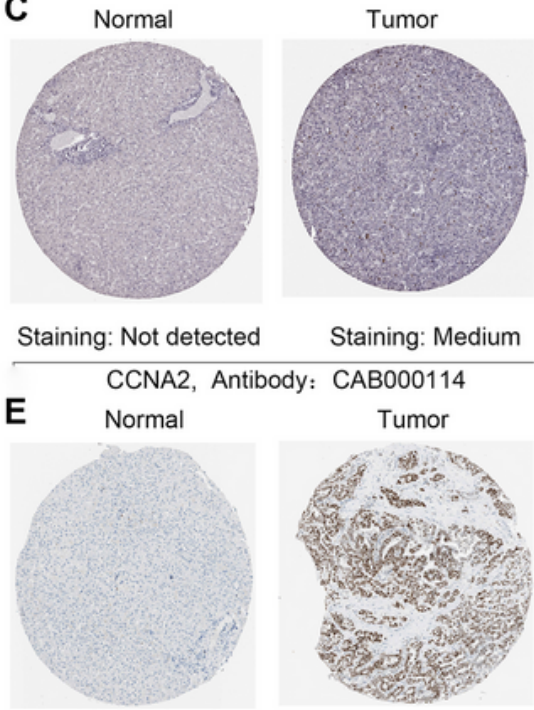

Staining: Medium
Staining: Not detected

G Normal

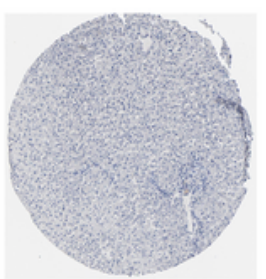

Staining: Not detected

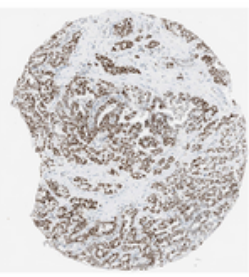

Staining: High
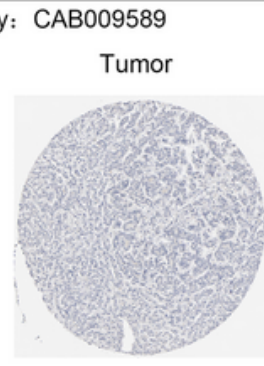

Staining: Medium

CDK1, Antibody: CAB003799

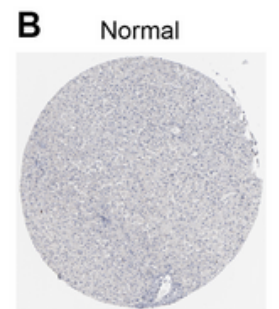

Staining: Not detected
Tumor

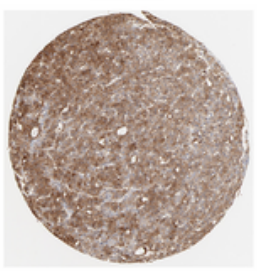

Staining: Medium

AURKA, Antibody: HPA002636

D
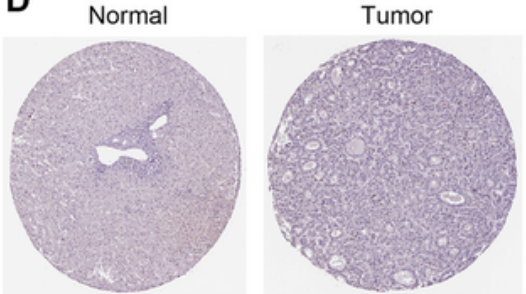

Staining: Not detected Staining: Medium

MKI67, Antibody: CAB000058

F Normal

Tumor
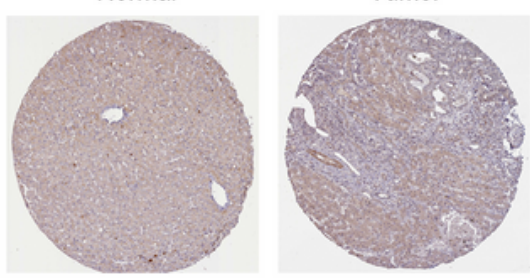

Staining: Low

Staining: Medium

CDC6, Antibody: HPA050114

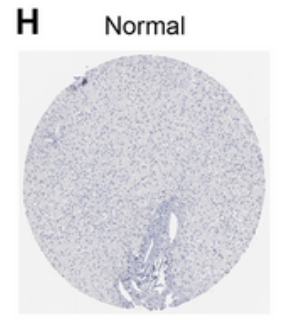

Tumor

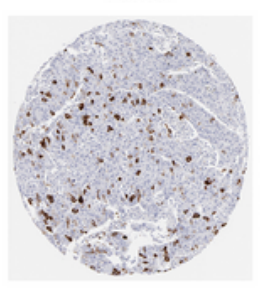

Staining: Medium

Staining: Not detected

CCNB1, Antibody: CAB003804

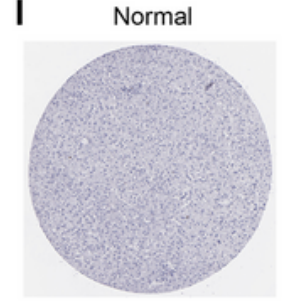

Staining: Not detected
Tumor

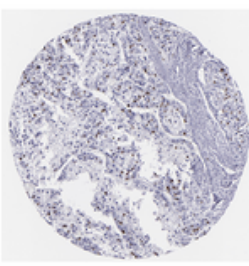

Staining: Medium

TOP2A, Antibody: CAB002448

\section{Figure 5}

Representative immunohistochemistry images of (A) FOXM1, (B) AURKA, (C) CCNA2, (D) MKI67, (E) EZH2, (F) CDC6, (G) CDK1, (H) CCNB1, and (I) TOP2A in HCC and non-cancerous liver tissues derived from the HPA database. HCC, hepatocellular carcinoma; HPA, Human Protein Atlas. 
A

B

Cancer types summary

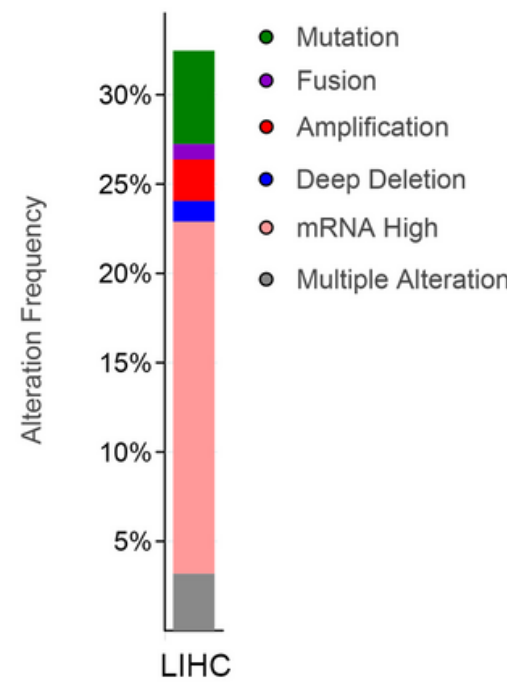

$\begin{array}{ll}\text { FOXM1 } & 7 \% \\ \text { AURKA } & 7 \% \\ \text { CCNA2 } & 2 \% \\ \text { CDKN3 } & 4 \% \\ \text { MK167 } & 3 \% \\ \text { EZH2 } & 8 \% \\ \text { CDC6 } & 8 \% \\ \text { CDK1 } & 5 \% \\ \text { CCNB1 } & 7 \% \\ \text { TOP2A } & 5 \%\end{array}$

Genetic Alteration

mRNA High No alterations

C

D
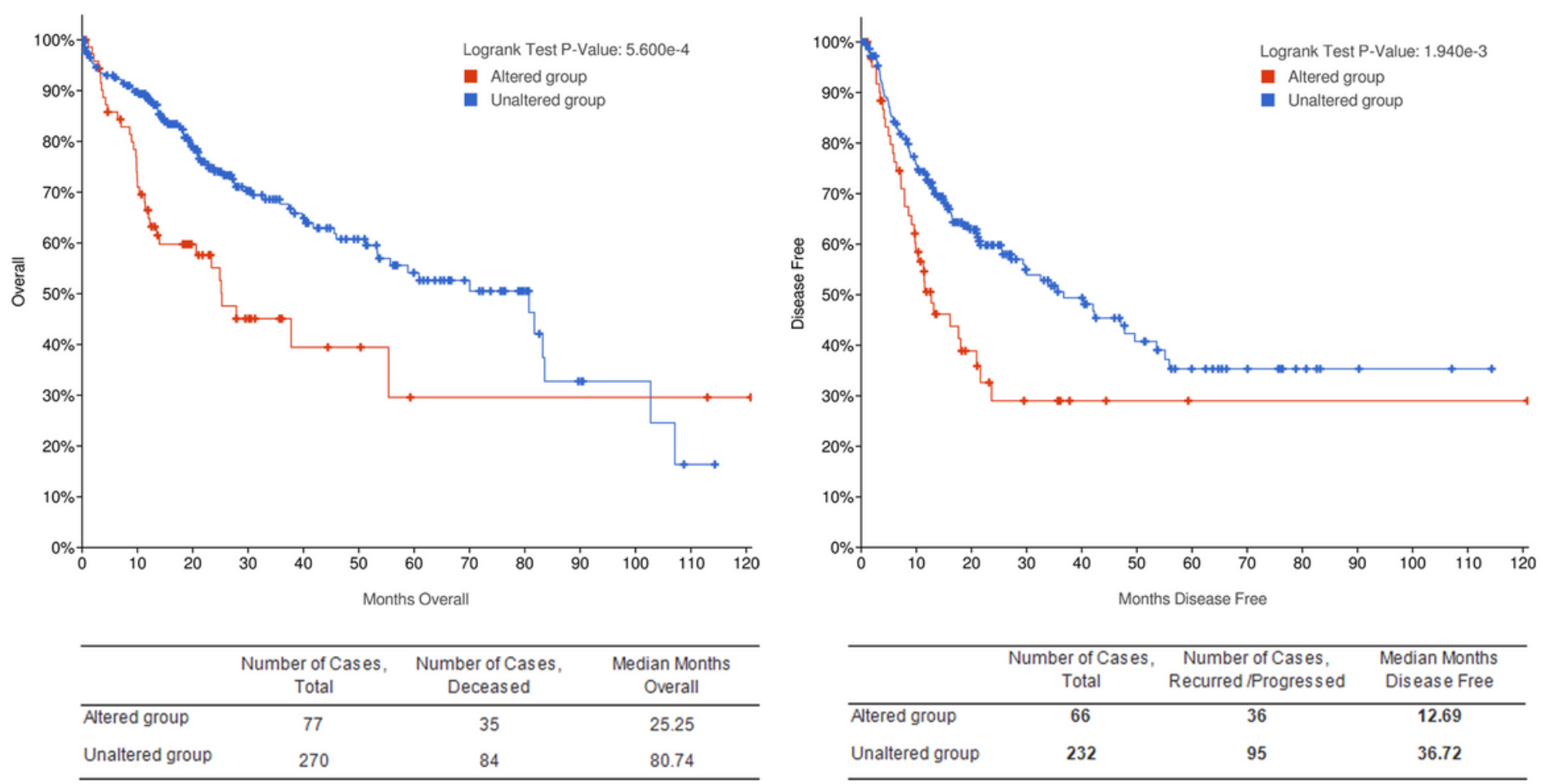

Figure 6

Alteration frequency and prognosis of the 10 hub genes. (A) The summary of the cancer types was used to calculate alteration frequency of the 10 hub genes in LIHC cases. (B) mRNA expression alterations (RNA Seq V2 RSEM) of the 10 hub genes in LIHC patients. (C) OS and (D) DFS/PFS of LIHC patients with altered (red) and unaltered (blue) mRNA expression of the 10 hub genes. LIHC, liver hepatocellular carcinoma; OS, overall survival; DFS/PFS, disease-free survival/progression-free survival. 
A

B

Cancer types summary

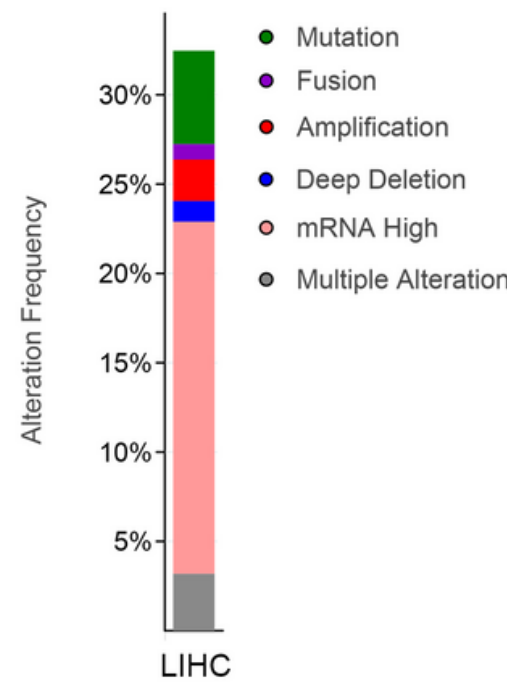

$\begin{array}{ll}\text { FOXM1 } & 7 \% \\ \text { AURKA } & 7 \% \\ \text { CCNA2 } & 2 \% \\ \text { CDKN3 } & 4 \% \\ \text { MK167 } & 3 \% \\ \text { EZH2 } & 8 \% \\ \text { CDC6 } & 8 \% \\ \text { CDK1 } & 5 \% \\ \text { CCNB1 } & 7 \% \\ \text { TOP2A } & 5 \%\end{array}$

Genetic Alteration

mRNA High No alterations

C

D
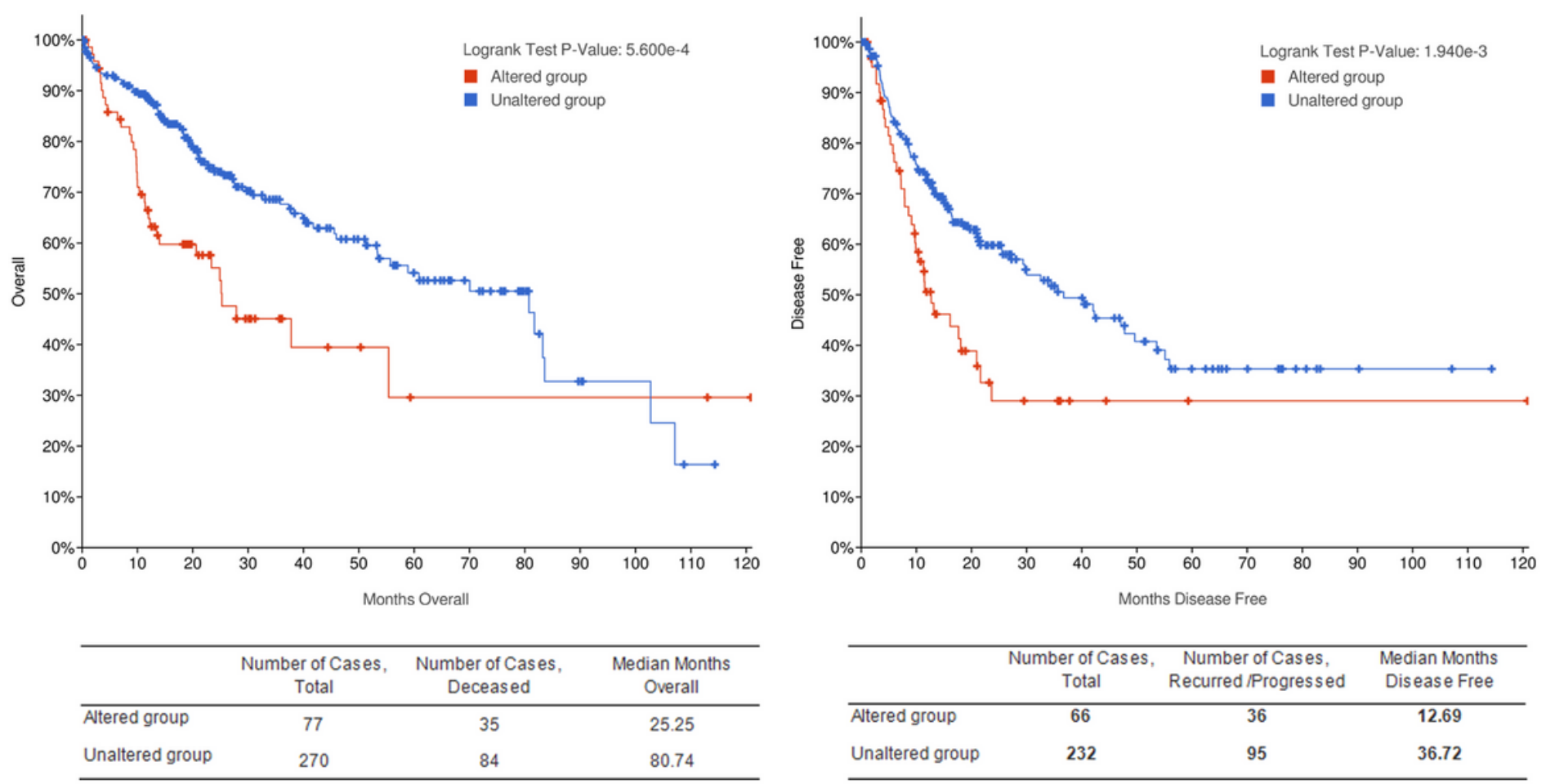

Figure 6

Alteration frequency and prognosis of the 10 hub genes. (A) The summary of the cancer types was used to calculate alteration frequency of the 10 hub genes in LIHC cases. (B) mRNA expression alterations (RNA Seq V2 RSEM) of the 10 hub genes in LIHC patients. (C) OS and (D) DFS/PFS of LIHC patients with altered (red) and unaltered (blue) mRNA expression of the 10 hub genes. LIHC, liver hepatocellular carcinoma; OS, overall survival; DFS/PFS, disease-free survival/progression-free survival. 
A

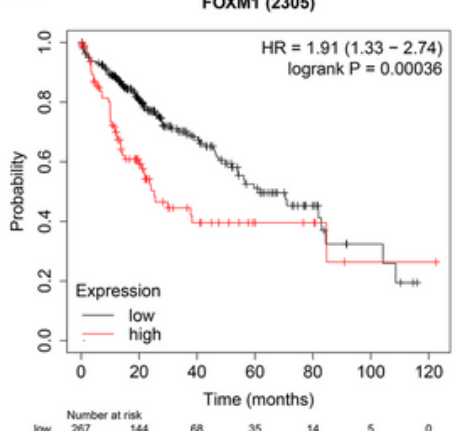

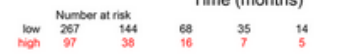

D

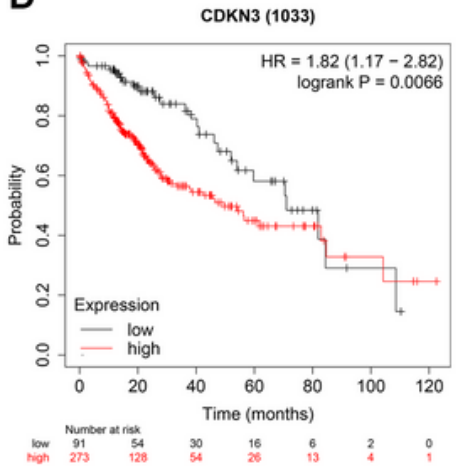

G

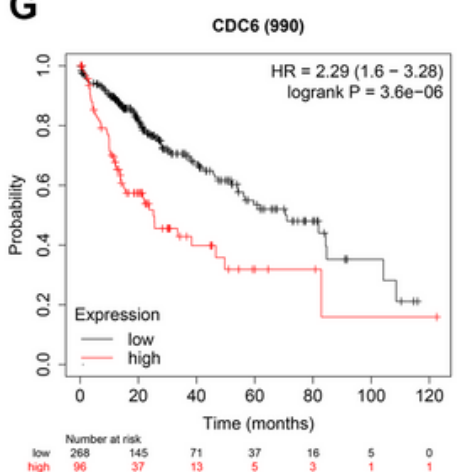

$$
\text { J }
$$

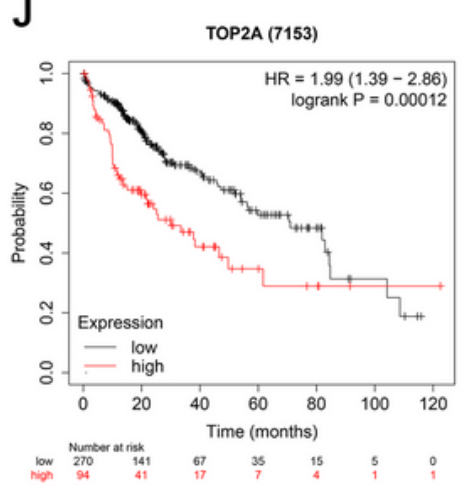

B

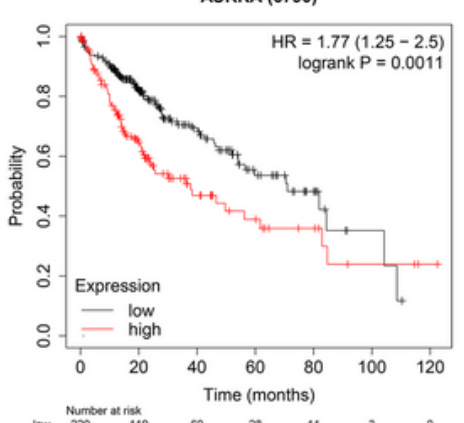

E

E

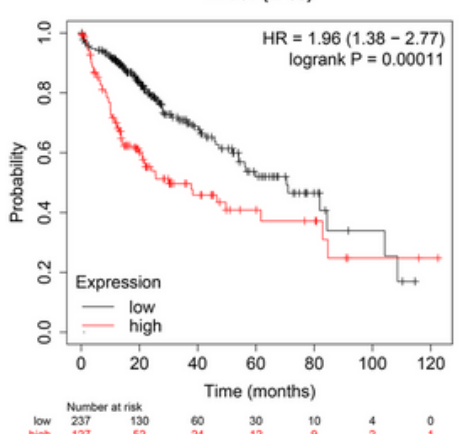

H

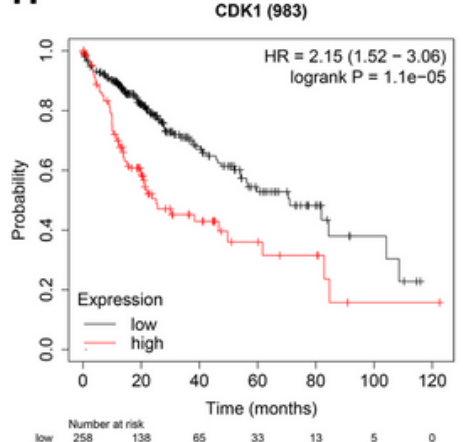

C

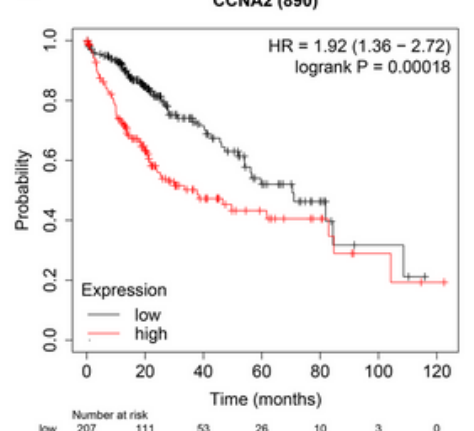

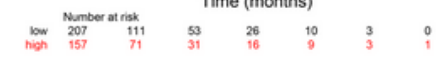

F

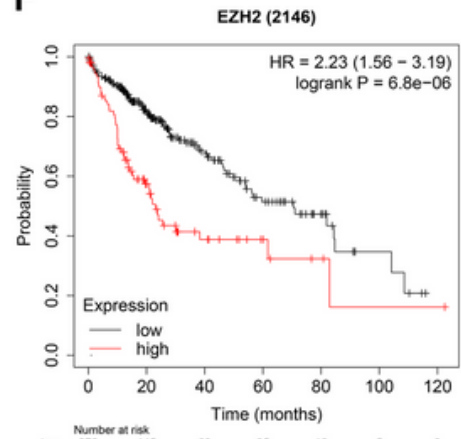

I

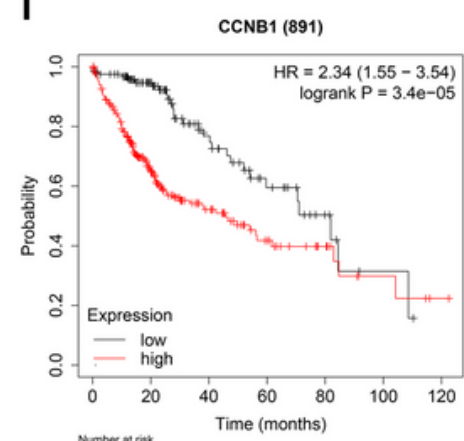

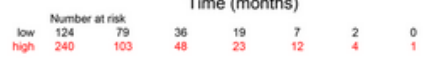

\section{Figure 7}

OS of the 10 hub genes overexpressed in patients with liver cancer was analyzed by Kaplan-Meier plotter. FOXM1, log-rank P=0.00036; AURKA, log-rank $P=0.0011$; CCNA2, log-rank $P=0.00018$; CDKN3, log-rank $P=0.0066 ;$ MKI67, log-rank $P=0.00011$; $E Z H 2$, log-rank $P=6.8 e-06 ; C D C 6$, log-rank $P=3.6 e-06$; CDK1, logrank $P=1.1$ e-05; CCNB1, log-rank $P=3.4 E-05$; and TOP2A, log-rank $P=0.00012$. Data are presented as Log- 
rank $\mathrm{P}$ and the hazard ratio with a $95 \%$ confidence interval. Log-rank $\mathrm{P}<0.01$ was regarded as statistically significant. OS, overall survival.

A

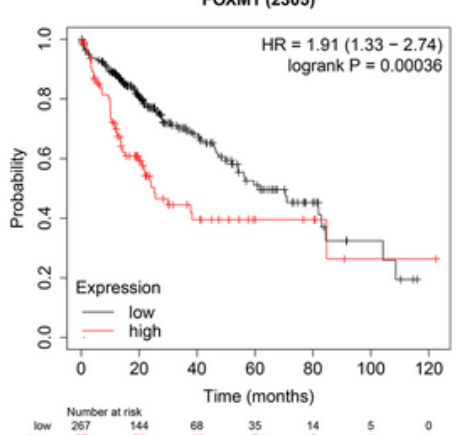

D
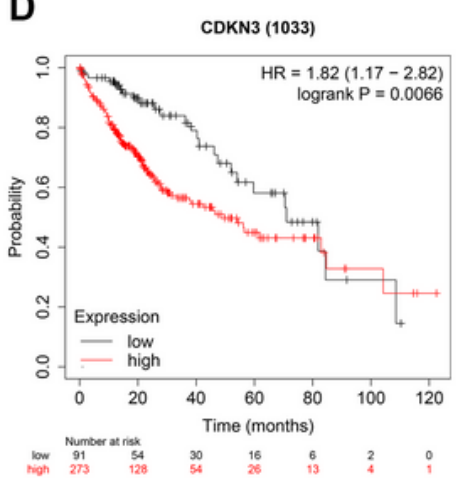

G

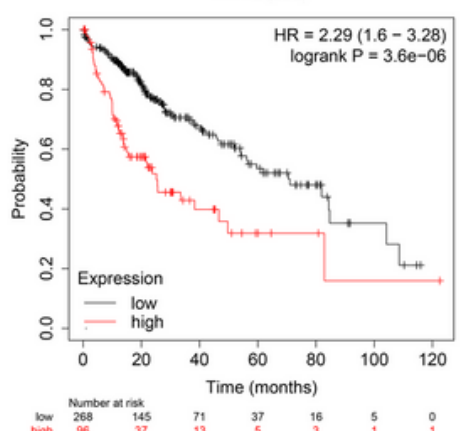

J

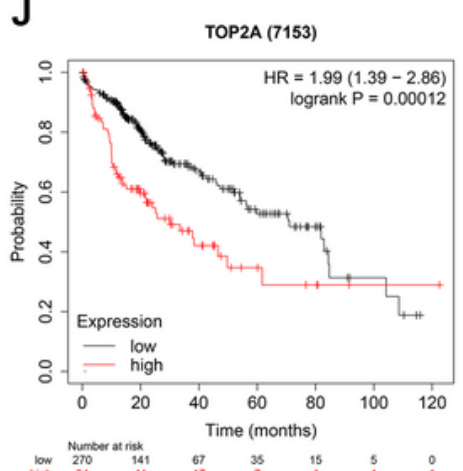

B

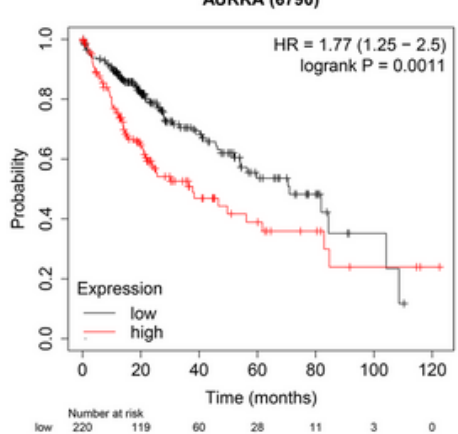

E

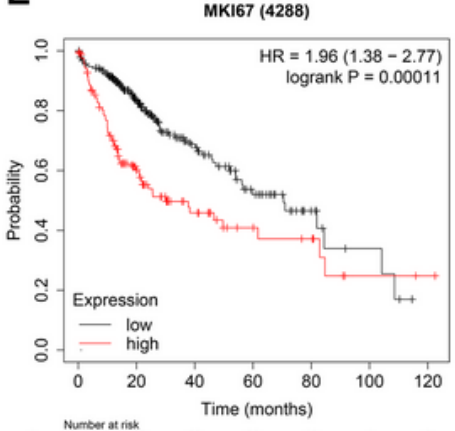

C
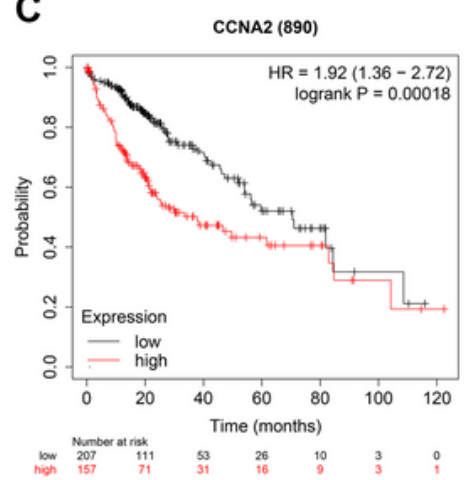

$\mathbf{F}$

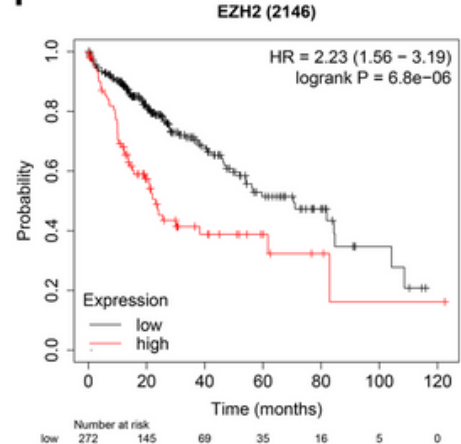

H

I CCNB1 (891)
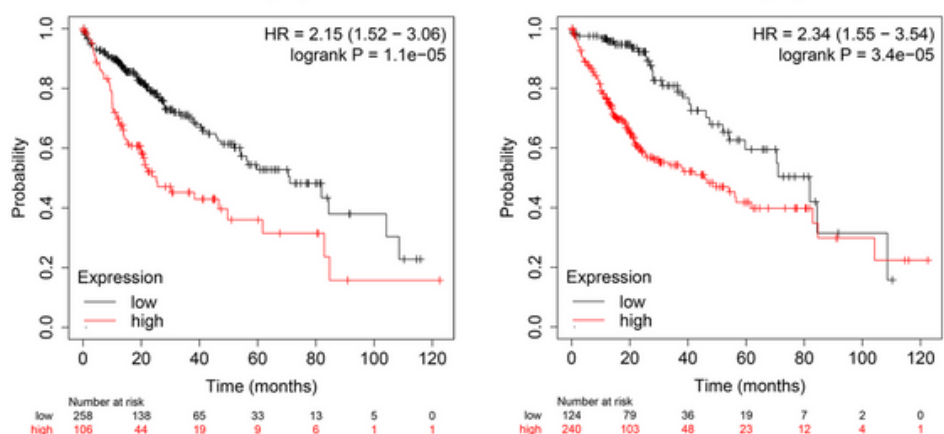

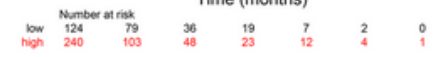

\section{Figure 7}

OS of the 10 hub genes overexpressed in patients with liver cancer was analyzed by Kaplan-Meier plotter. FOXM1, log-rank P=0.00036; AURKA, log-rank P=0.0011; CCNA2, log-rank P=0.00018; CDKN3, log-rank $\mathrm{P}=0.0066 ;$ MKI67, log-rank P=0.00011; EZH2, log-rank P=6.8e-06; CDC6, log-rank P=3.6e-06; CDK1, log- 
rank $P=1.1 \mathrm{e}-05$; $C C N B 1$, log-rank $P=3.4 \mathrm{E}-05$; and TOP2A, log-rank $P=0.00012$. Data are presented as Logrank $P$ and the hazard ratio with a $95 \%$ confidence interval. Log-rank $P<0.01$ was regarded as statistically significant. OS, overall survival.
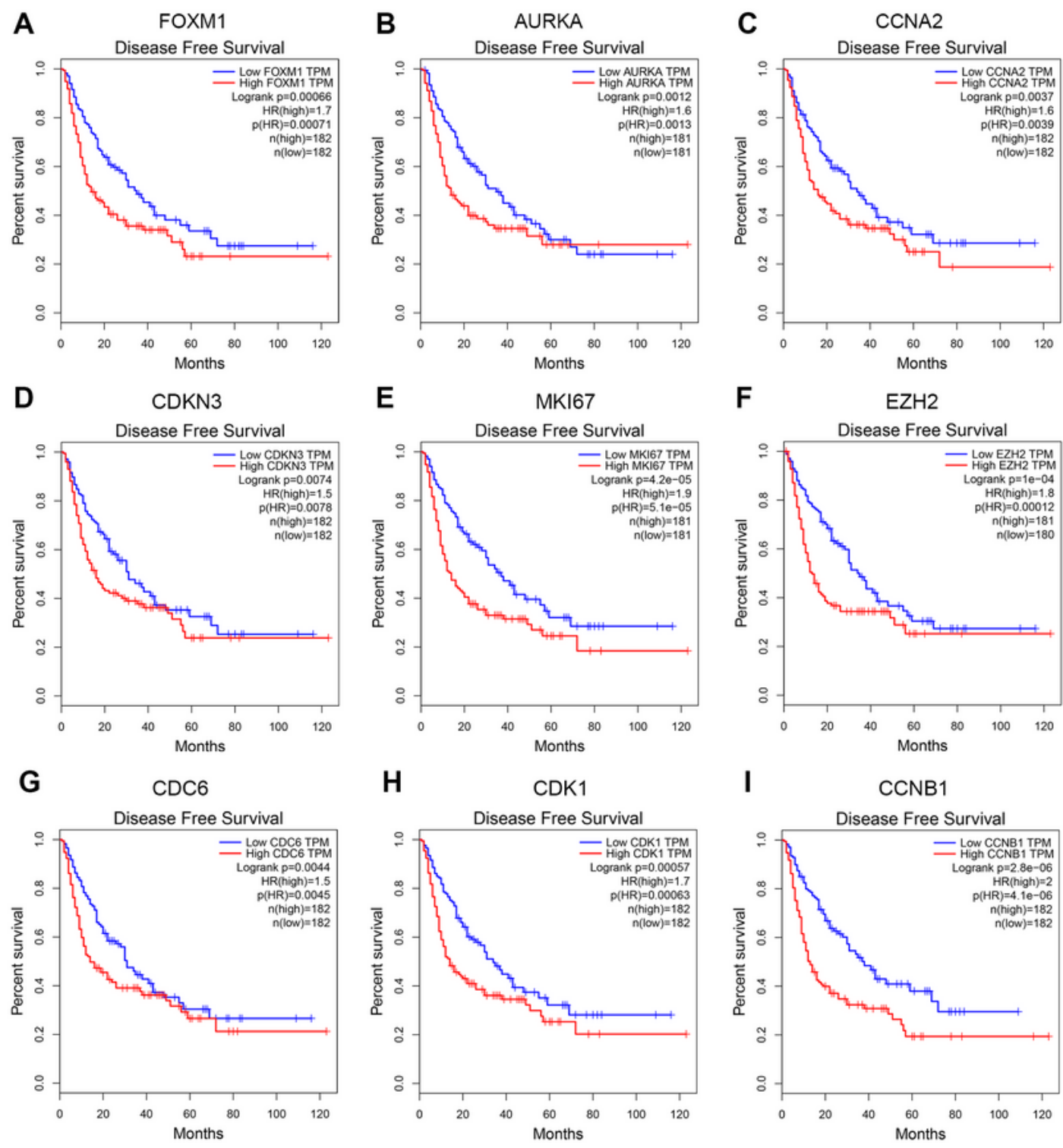

I
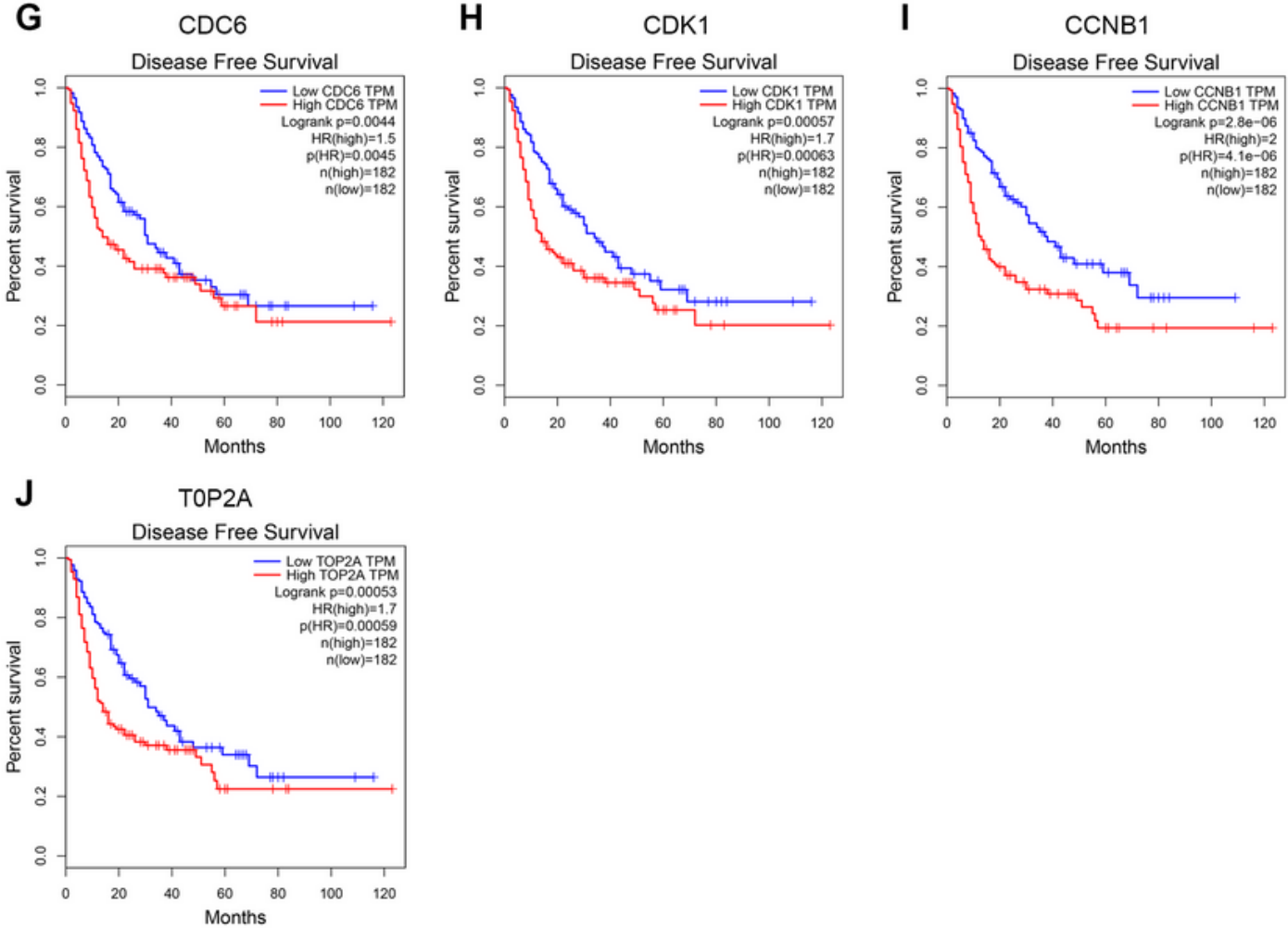

Figure 8

DFS of LIHC patients overexpressed the 10 hub genes were analyzed by the GEPIA online database. Data are presented as log-rank P and the hazard ratio with a 95\% confidence interval. FOXM1, log-rank 
$P=0.00066$; AURKA, log-rank $P=0.0012 ; C C N A 2$, log-rank $P=0.0037 ; C D K N 3$, log-rank $P=0.0074 ; M K I 67$, log-rank $P=4.2 e-05 ; E Z H 2$, log-rank $P=1 e-04 ; C D C 6$, log-rank $P=0.0044 ; C D K 1$, log-rank $P=0.00057 ; C C N B 1$, log-rank $P=2.8 E-06$; and TOP2A, log-rank $P=0.00053$. Log-rank $P<0.01$ was considered statistically significant. DFS, disease-free survival; LIHC, liver hepatocellular carcinoma.
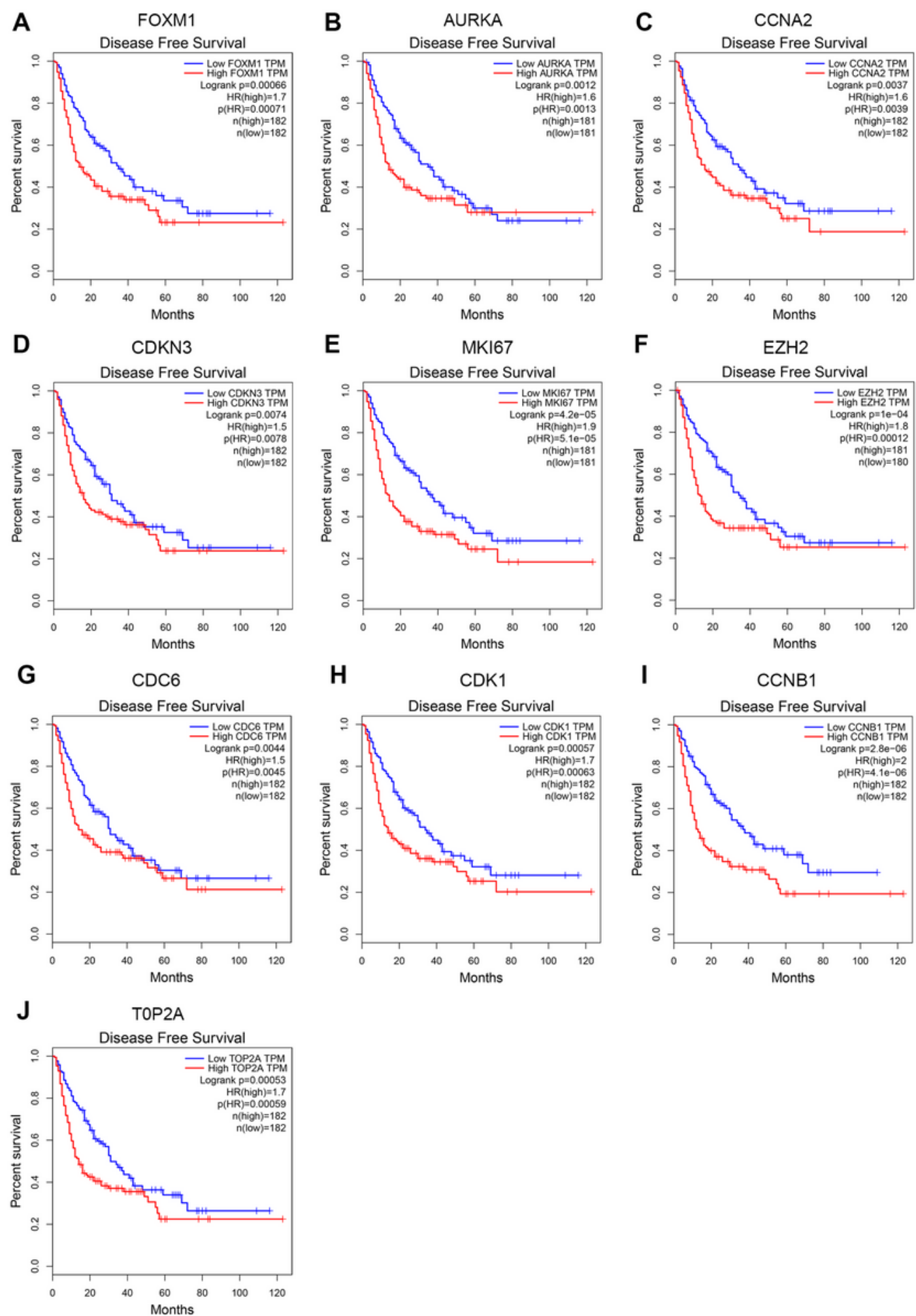

Figure 8 
DFS of LIHC patients overexpressed the 10 hub genes were analyzed by the GEPIA online database. Data are presented as log-rank $P$ and the hazard ratio with a 95\% confidence interval. FOXM1, log-rank $P=0.00066$; AURKA, log-rank $P=0.0012 ; C C N A 2$, log-rank $P=0.0037$; $C D K N 3$, log-rank $P=0.0074 ;$ MKI67, log-rank $P=4.2 e-05 ; E Z H 2$, log-rank $P=1 e-04 ; C D C 6$, log-rank $P=0.0044 ; C D K 1$, log-rank $P=0.00057$; CCNB1, log-rank $P=2.8 E-06$; and TOP2A, log-rank $P=0.00053$. Log-rank $P<0.01$ was considered statistically significant. DFS, disease-free survival; LIHC, liver hepatocellular carcinoma.

\section{Supplementary Files}

This is a list of supplementary files associated with this preprint. Click to download.

- Tables1.xls

- Tables1.xls

- Figure.S7.png

- Figure.S7.png

- Figure.S6.png

- Figure.S6.png

- Figure.S5.png

- Figure.S5.png

- Figure.S4.png

- Figure.S4.png

- Figure.S3.tif

- Figure.S3.tif

- Figure.S2.tif

- Figure.S2.tif

- Figure.S1.tif

- Figure.S1.tif 